\title{
Nosology and Classification of Genetic Skeletal Disorders: 2010 Revision
}

\section{Matthew L. Warman, ${ }^{1}$ Valerie Cormier-Daire, ${ }^{2}$ Christine Hall, ${ }^{3}$ Deborah Krakow, ${ }^{4,5}$ Ralph Lachman, ${ }^{4}$ Martine LeMerrer, ${ }^{2}$ Geert Mortier, ${ }^{6}$ Stefan Mundlos, ${ }^{7}$ Gen Nishimura, ${ }^{8}$ David L. Rimoin, ${ }^{4}$ Stephen Robertson, ${ }^{9}$ Ravi Savarirayan, ${ }^{10}$ David Sillence, ${ }^{11}$ Juergen Spranger, ${ }^{12}$ Sheila Unger, ${ }^{12,13}$ Bernhard Zabel, ${ }^{12}$ and Andrea Superti-Furga ${ }^{12,14 *}$}

${ }^{1}$ Orthopaedic Research Laboratories, Department of Orthopaedic Surgery, The Howard Hughes Medical Institute, Children's Hospital, Boston, Massachusetts

${ }^{2}$ Department of Genetics and INSERM U781, Paris Descartes University, Hôpital Necker Enfants Malades, Paris, France

${ }^{3}$ Institute of Child Health, University of London, London, UK

${ }^{4}$ Medical Genetics Institute, Steven Spielberg Building, Cedars-Sinai Medical Center, Los Angeles, California

${ }^{5}$ Departments of Orthopaedic Surgery and Human Genetics, UCLA, Los Angeles, California

${ }^{6}$ Department of Medical Genetics, University Hospital of Antwerp, University of Antwerp, Edegem, Belgium

'Institut für Medizinische Genetik, Charité Universitätsmedizin Berlin, Max-Planck-Institut für Molekulare Genetik, Berlin, Germany

${ }^{8}$ Department of Pediatric Imaging, Tokyo Metropolitan Children's Medical Center, Fuchu, Tokyo, Japan

${ }^{9}$ Department of Paediatrics and Child Health, Dunedin School of Medicine, Otago University, Dunedin, New Zealand

${ }^{10}$ Murdoch Children's Research Institute, Royal Children's Hospital, Department of Paediatrics, University of Melbourne, Victoria, Australia

${ }^{11}$ Discipline of Genetic Medicine, The Children's Hospital at Westmead Clinical School, The University of Sydney, Westmead, Australia

${ }^{12}$ Centre for Pediatrics and Adolescent Medicine, Freiburg University Hospital, University of Freiburg, Freiburg, Germany

${ }^{13}$ Medical Genetics Service, University of Lausanne, CHUV-Centre Hospitalier Universitaire Vaudois, Lausanne, Switzerland

${ }^{14}$ Department of Pediatrics, University of Lausanne, CHUV-Centre Hospitalier Universitaire Vaudois, Lausanne, Switzerland

Received 16 December 2010; Accepted 30 December 2010

Genetic disorders involving the skeletal system arise through disturbances in the complex processes of skeletal development, growth and homeostasis and remain a diagnostic challenge because of their variety. The Nosology and Classification of Genetic Skeletal Disorders provides an overview of recognized diagnostic entities and groups them by clinical and radiographic features and molecular pathogenesis. The aim is to provide the Genetics, Pediatrics and Radiology community with a list of recognized genetic skeletal disorders that can be of help in the

How to Cite this Article:

Warman ML, Cormier-Daire V, Hall C, Krakow D, Lachman R, LeMerrer M, Mortier G, Mundlos S, Nishimura G, Rimoin DL, Robertson S, Savarirayan R, Sillence D, Spranger J, Unger S, Zabel B, Superti-Furga A. 2011. Nosology and classification of genetic skeletal disorders: 2010 revision.

Am J Med Genet Part A 155:943-968. diagnosis of individual cases, in the delineation of novel disorders, and in building bridges between clinicians and scientists interested in skeletal biology. In the 2010 revision, 456 conditions were included and placed in 40 groups defined by molecular, biochemical, and/or radiographic criteria. Of these conditions, 316 were associated with mutations in one or more of 226 different genes, ranging from common, recurrent mutations to "private" found in single families or individuals. Thus, the Nosology is a hybrid between a list of clinically defined

The 9th ISDS meeting and the Nosology workshop were held in Boston in July 2009 and supported by The Manton Center for Orphan Disease Research, Children's Hospital, Boston, Massachusetts; Children's Orthopaedic Surgery Foundation, Inc., Boston, Massachusetts; The Osteogenesis Imperfecta Foundation, Gaithersburg, Maryland; Biomarin, Novato, California; and Enobia Pharma, Montreal, Quebec, Canada. The 2010 Nosology tables are available online at the International Skeletal Dysplasia Society web site (www.isds.ch).

${ }^{\star}$ Correspondence to:

Andrea Superti-Furga, Centre Hospitalier Universitaire Vaudois (CHUV), Av. Decker, 2, 1011 Lausanne, Switzerland. E-mail: asuperti@unil.ch Published online 15 March 2011 in Wiley Online Library (wileyonlinelibrary.com).

DOI 10.1002/ajmg.a.33909 
disorders, waiting for molecular clarification, and an annotated database documenting the phenotypic spectrum produced by mutations in a given gene. The Nosology should be useful for the diagnosis of patients with genetic skeletal diseases, particularly in view of the information flood expected with the novel sequencing technologies; in the delineation of clinical entities and novel disorders, by providing an overview of established nosologic entities; and for scientists looking for the clinical correlates of genes, proteins and pathways involved in skeletal biology. (ㄷ) 2011 Wiley-Liss, Inc.

Key words: skeletal genetics; osteochondrodysplasias; nosology; dysostoses; molecular basis of disease

\section{INTRODUCTION}

In the 1960s, accumulating evidence that genetic skeletal disorders were clinically and genetically heterogeneous prompted a group of international experts to prepare a document to reach an agreement on the nomenclature of what was then called "constitutional (or intrinsic) disorders of bone" [1970, 1971a,b,c,d; McKusick and Scott, 1971]. The "Nomenclature" was meant to bring together experts in radiology, clinical genetics, and pediatrics to agree on the denomination and classification of skeletal disorders, syndromes and metabolic diseases that were being newly described. Revisions have been prepared in 1977, 1983, 1992, and 1997 [1978, 1979, 1983, 1998, Rimoin, 1979; Spranger, 1992; Lachman, 1998]. Following the establishment of the International Skeletal Dysplasia Society (ISDS) in 1999, and to cope with the increasing complexity of information, revisions of the Nosology have been delegated to an expert group nominated ad hoc within the ISDS to ensure an adequate representation of clinical, radiological and molecular expertise (2001 and 2006 revisions) [Hall, 2002; Superti-Furga and Unger, 2007].

\section{METHODS}

The Nosology Group of the International Skeletal Dysplasia Society met in August 2009. A consensus was reached for changes to be made to the grouping of disorders and about the inclusion of individual disorders. The drafts were circulated after the meeting and an effort was made to monitor recent publications up to November 2010. The criteria used for inclusion of individual disorders were unchanged from the previous revision. They were:

(1) Significant skeletal involvement, corresponding to the definition of skeletal dysplasias, metabolic bone disorders, dysostoses, and skeletal malformation and/or reduction syndromes.

(2) Publication and/or listing in MIM (meaning that observations should not find their way into the Nosology before they achieve peer-reviewed publication status).

(3) Genetic basis proven by pedigree or very likely based on homogeneity of phenotype in unrelated families.

(4) Nosologic autonomy confirmed by molecular or linkage analysis and/or by the presence of distinctive diagnostic features and of observation in multiple individuals or families.

\section{RESULTS}

Four hundred fifty-six different conditions were included and placed in 40 groups defined by molecular, biochemical and/or radiographic criteria. Of these conditions, 316 (2006 revision: 215 ) were associated with one or more of 226 (2006 revision: 140) different genes. The results are presented in Table I. Within a group, disorders with known molecular basis have been listed preceding those with lesser degree of evidence; however, variants of the same disorder have been kept together.

The organization of groups has been further changed in comparison to the 2006 version. Two new groups based on a common affected molecule or biochemical pathway have been created (TRPV4 group and Aggrecan group). The TRPV4 group includes disorders that are relatively common and that constitute a new prototypic spectrum ranging from mild to lethal. Aggrecan is one of the important structural molecules in cartilage and it would not be surprising if more disorders would find their way into this group in the future. Thus, groups 1-8 are based on a common underlying gene or pathway.

Groups 9-17 are based on the localization of radiographic changes to specific bone structures (vertebrae, epiphyses, metaphyses, diaphysis, or combination thereof) or of the involved segment (rhizo, meso, or acro). Groups 18-20 are defined by macroscopic criteria in combination with clinical features (bent bones, slender bones, presence of multiple dislocations). Groups 21-25 and 28 take into account features of mineralization (increased or reduced bone density, impaired mineralization, stippling, osteolysis). Group 27 encompasses the large group of lysosomal disorders with skeletal involvement. Group 29 comprises disorders with so-called abnormal (previously "anarchic") development of skeletal components such as exostoses, ecnhondromas, and ectopic calcification. It is particularly heterogeneous and may need to be revised in the future with the help of newer molecular data.

Group 23, comprising the osteopetrosis (OP) variants and related disorders, has been expanded following the identification of distinct genetic defects in various variants of osteopetrosis. The diversity of molecular mechanisms involved and the presence of clinical, biochemical and/or histologic features that distinguish between the various OP forms justify the subdivision of the "OP phenotype" in the many subtypes.

Group 25 (Osteogenesis Imperfecta and decreased bone density group) has had special attention. The Sillence classification, published 30 years ago, provided a first systematic clinical classification and made correlations to the inheritance pattern of individual clinical types [Sillence and Rimoin, 1978; Sillence et al., 1979a,b]. Today, a surprising genetic complexity of the molecular bases of OI has been revealed, and at the same time the extensive phenotypic variation arising from single loci has been documented clearly. It seemed therefore untenable to try and maintain tight correlations between "Sillence types" and their molecular basis. It was agreed upon to retain the Sillence classification as the prototypic and universally accepted way to classify the degree of severity in OI; and to free the Sillence classification from any direct molecular reference. Thus, the many genes that may cause osteogenesis imperfecta have been listed separately. The proliferation of "OI types" to reflect 


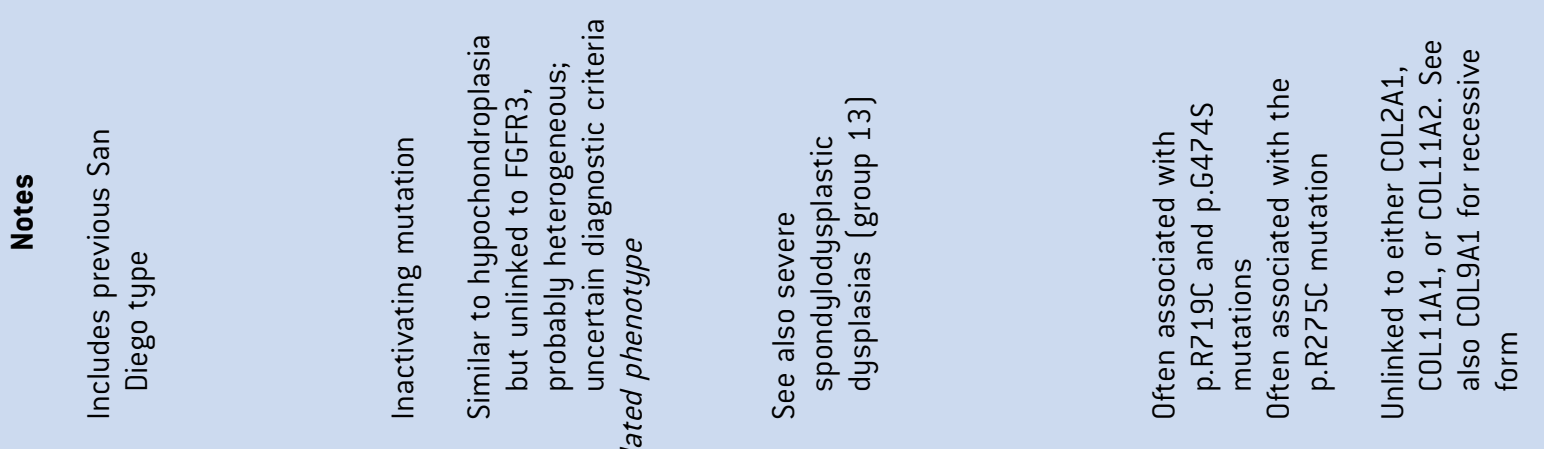

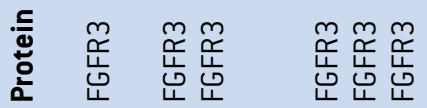

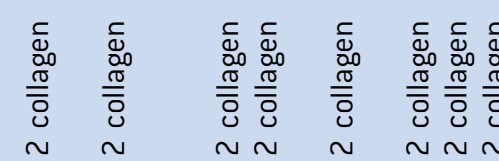

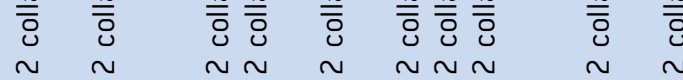

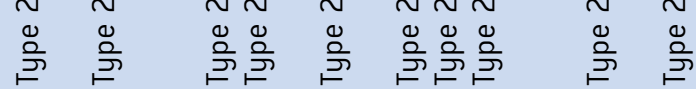

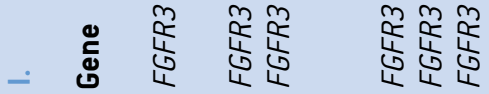

ปร

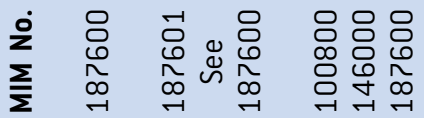

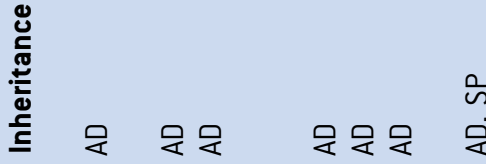

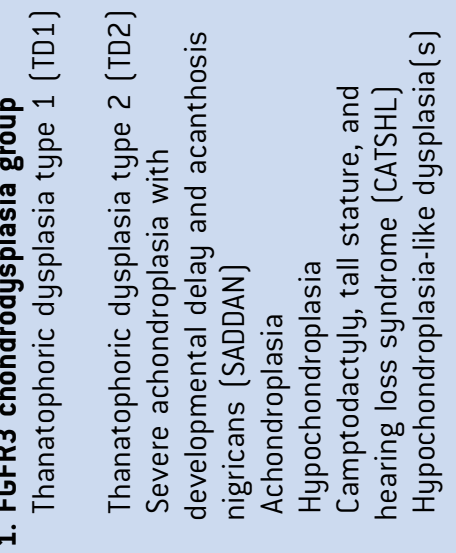

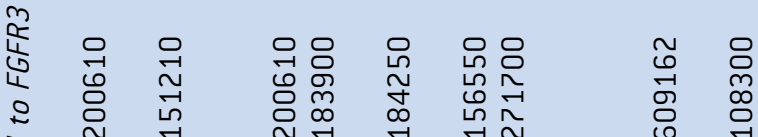

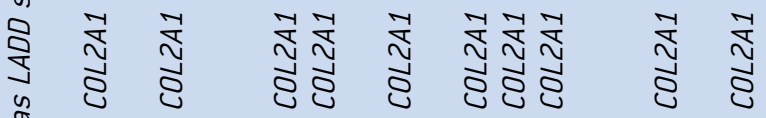
$\stackrel{2}{\infty}$

is

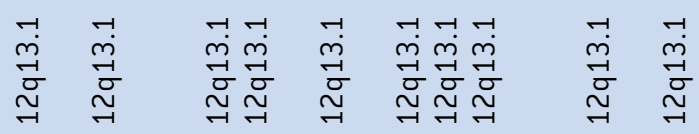

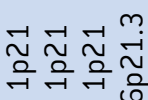

$\stackrel{2}{\stackrel{2}{\infty}}$

亭星是是是是是是是是是是

是是爱爱

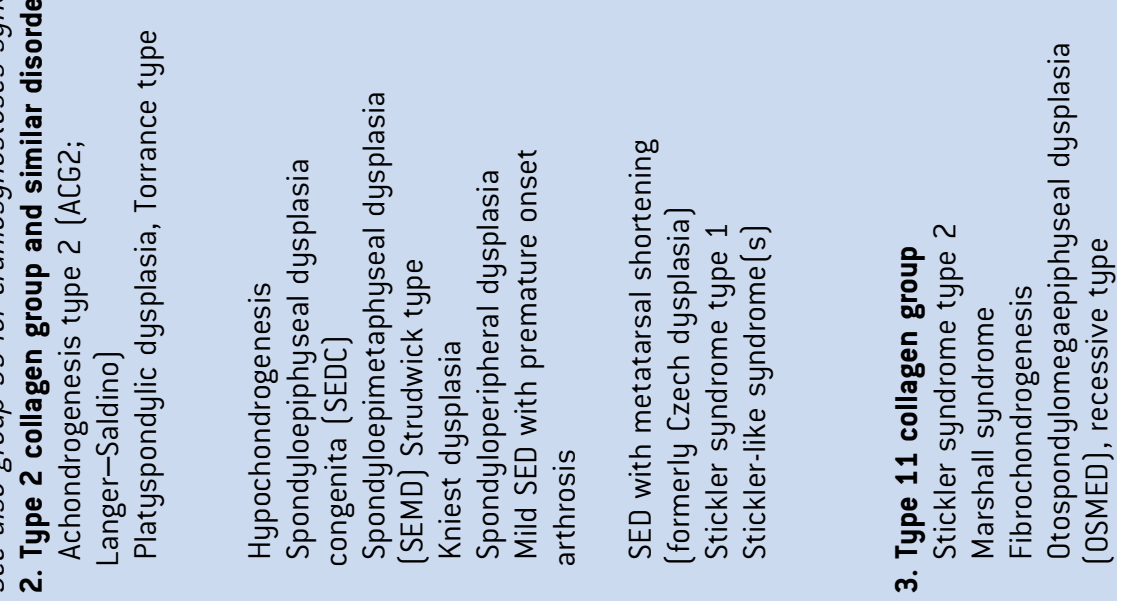



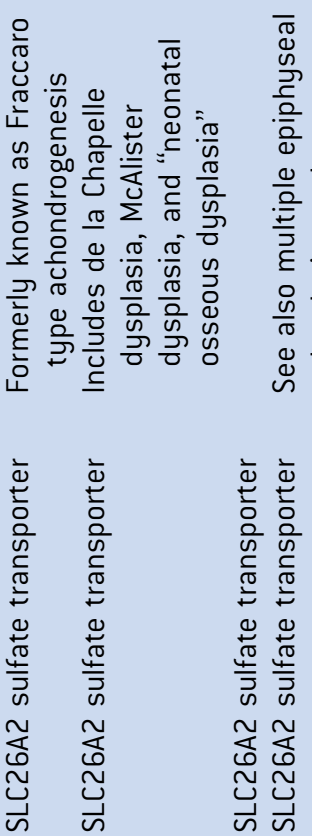
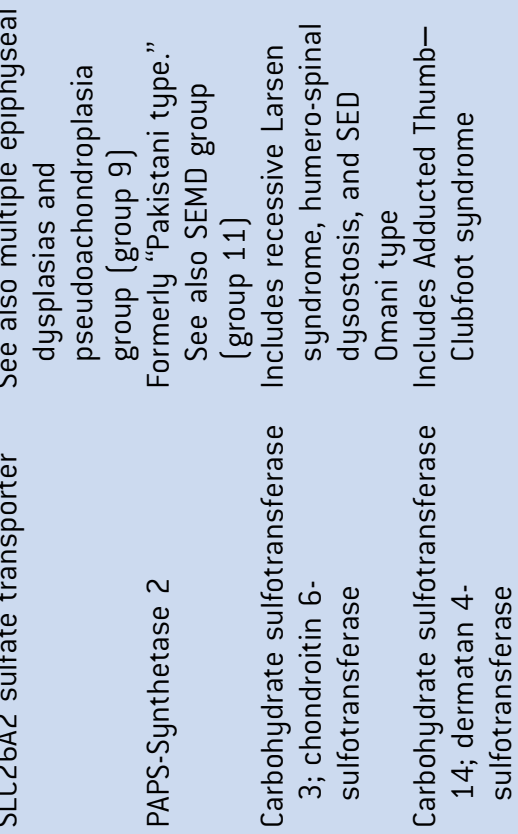

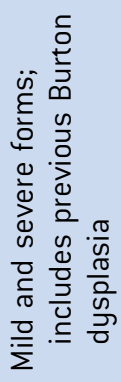

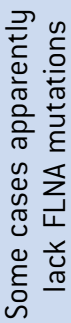
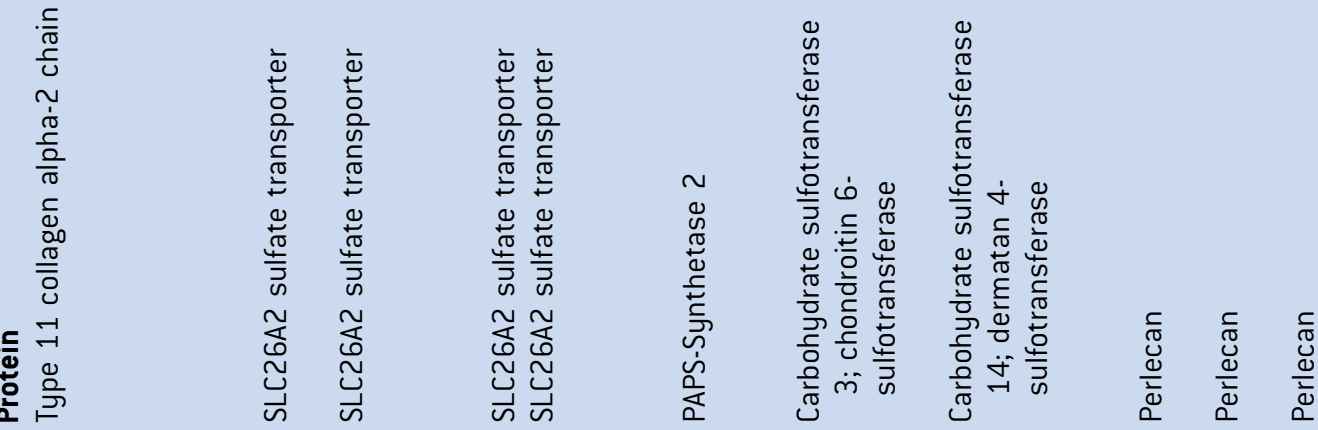

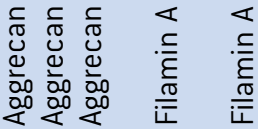

离

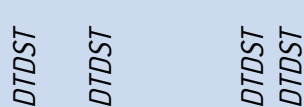

崩 竞

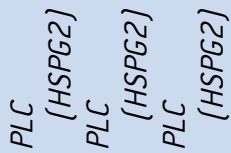

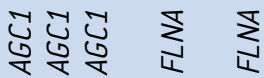

岂

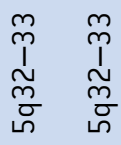

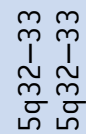

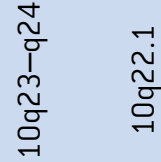

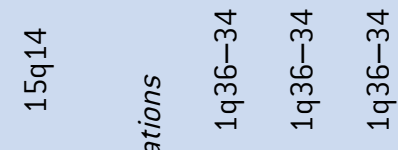

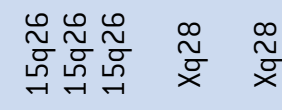

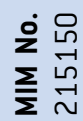

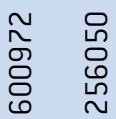

$\circ$
$\stackrel{0}{0}$
$\stackrel{0}{\sim}$
$\sim$

$\begin{array}{ll}n & \tilde{O} \\ 0 & 0 \\ \tilde{m} & 0 \\ 0 & 0 \\ 0 & 0\end{array}$

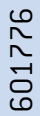

है

巡

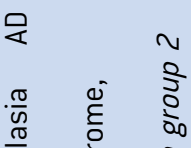
兴 爰爰

产爱 兴 妥
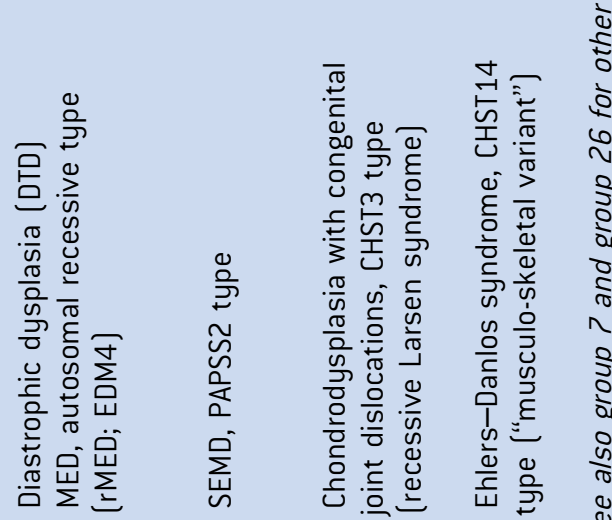

象

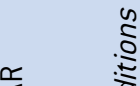

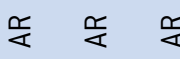
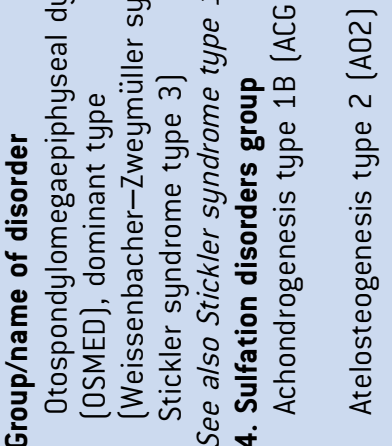

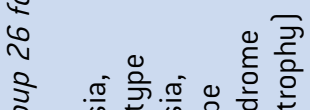

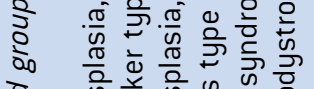
के क्षे

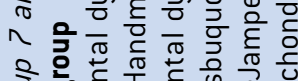
รั.

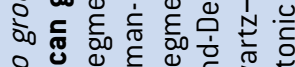

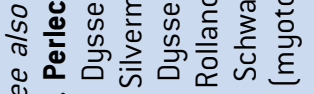

是妥是离号

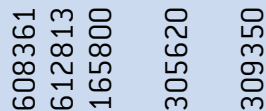

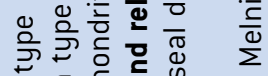

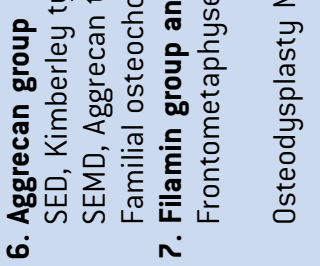



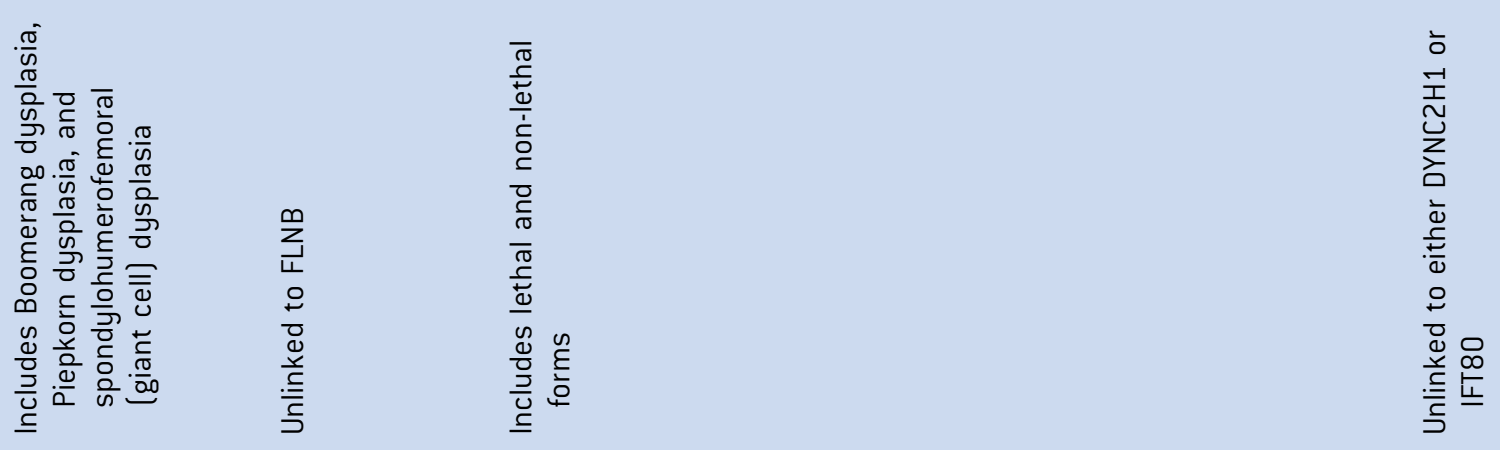

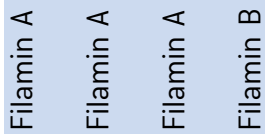
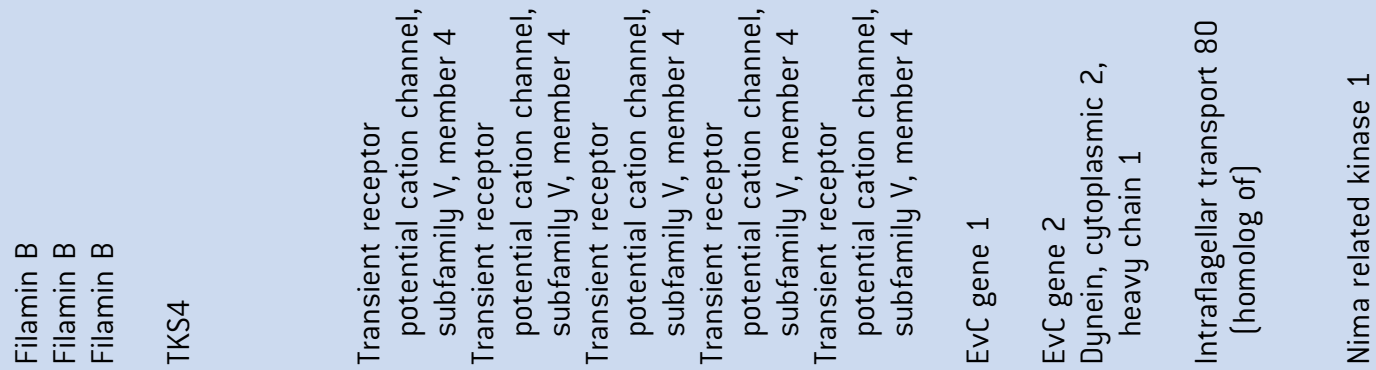

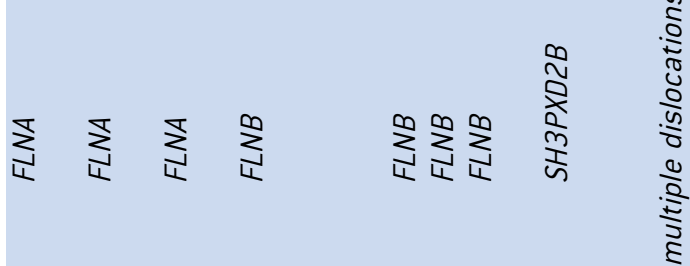

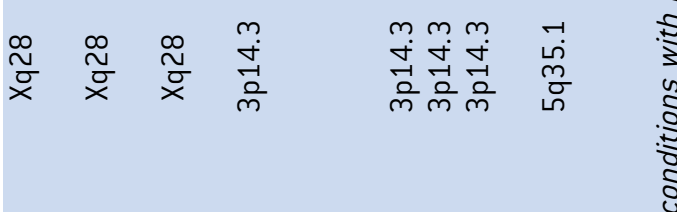

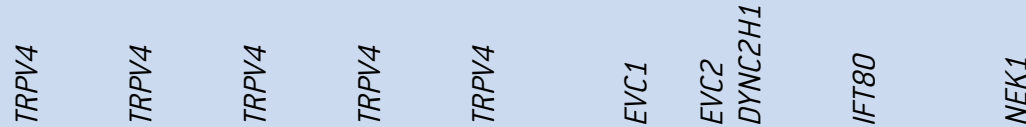

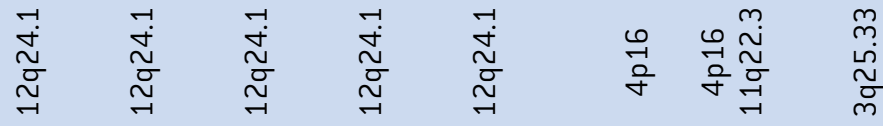

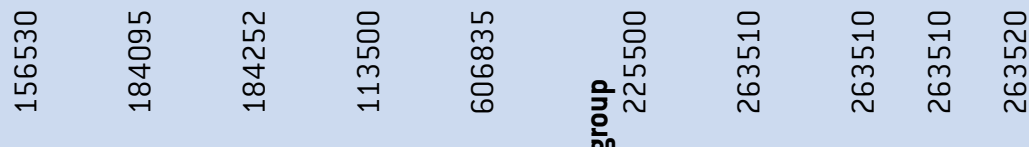

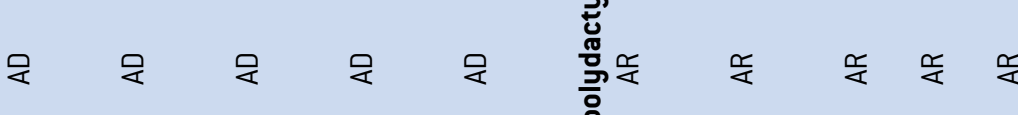

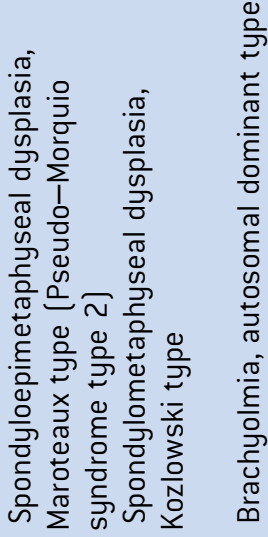

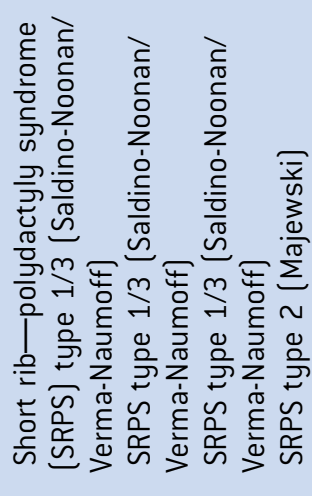




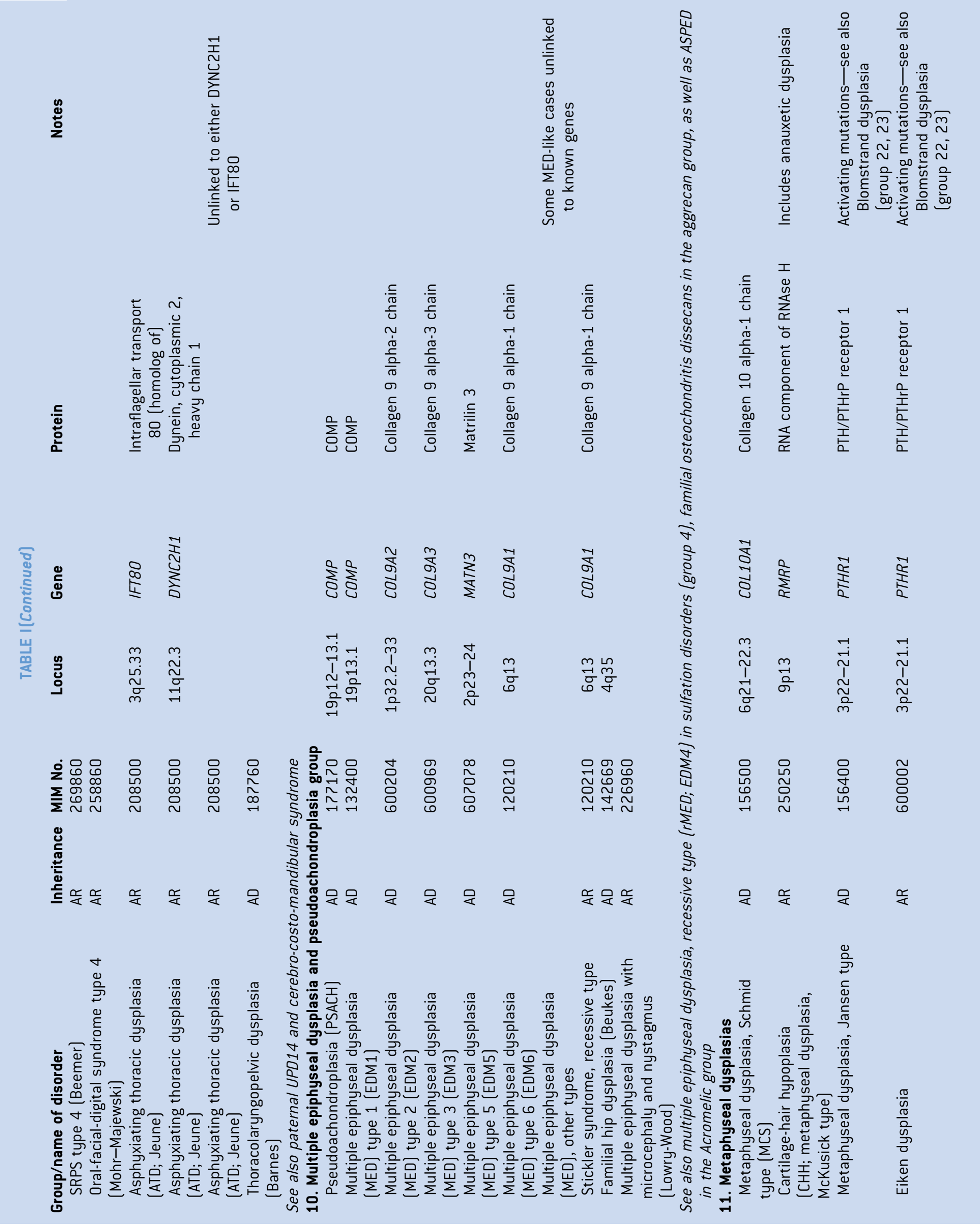




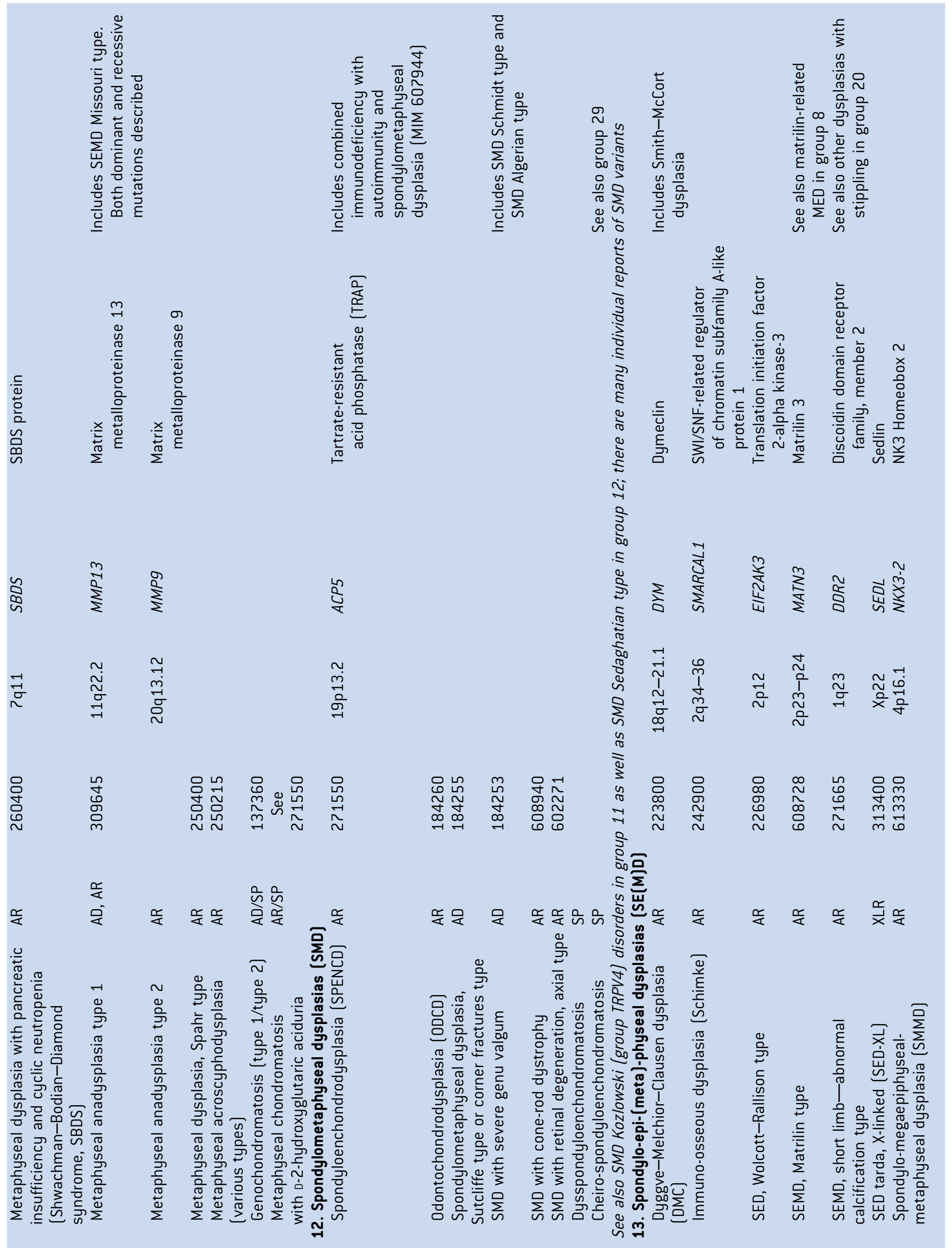




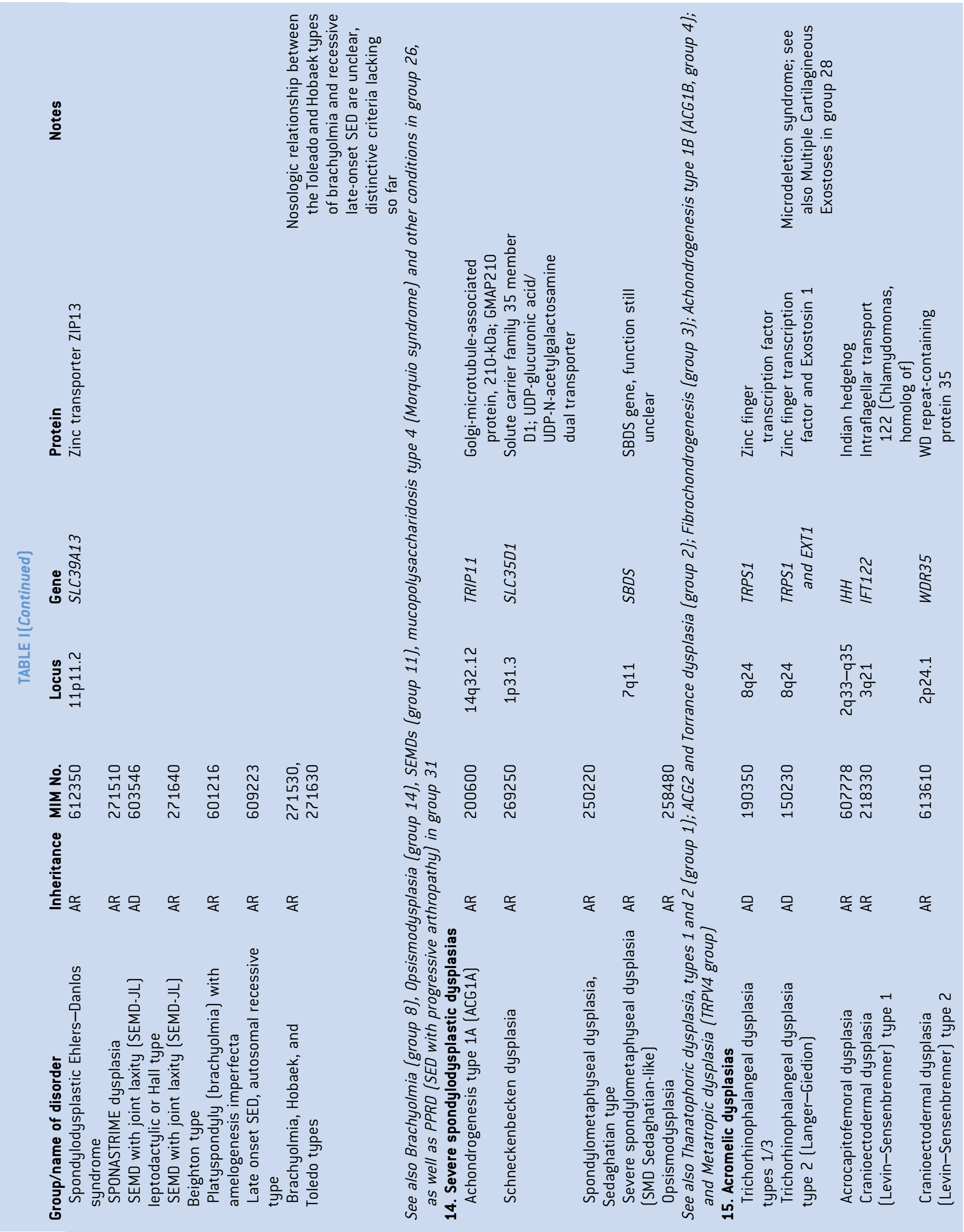




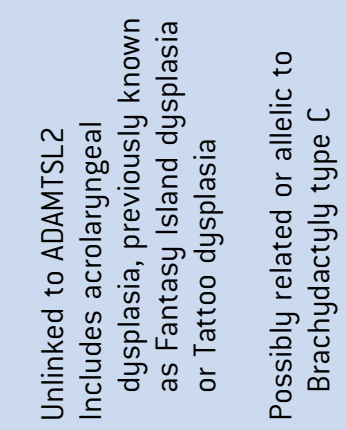

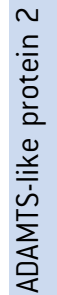

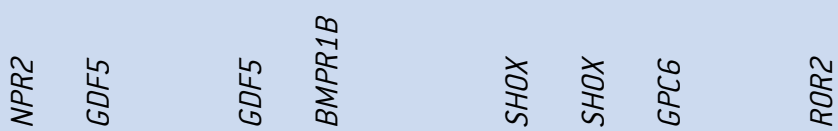

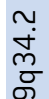

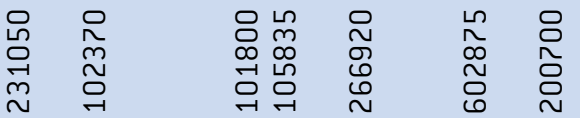

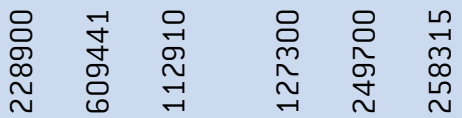

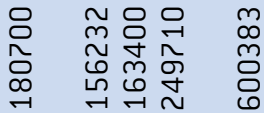

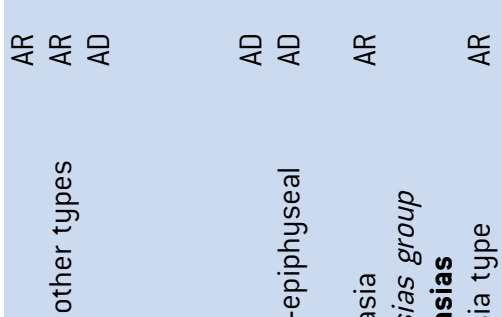

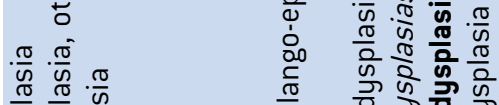

㐫 $\frac{\pi}{n} \frac{\pi}{n}$

गे ज़

늠 $\frac{u}{n} \cdot \frac{u}{2}$

등 응 응

过 $\frac{0}{0}$

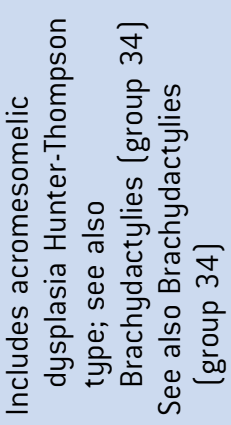

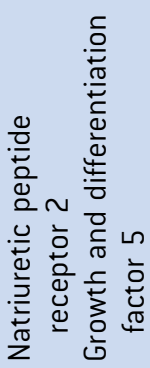

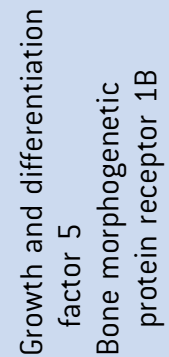

$\underset{\substack{\infty \\ \infty \\ 0 \\ \sim}}{\sim}$

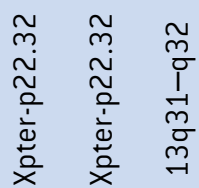

กั

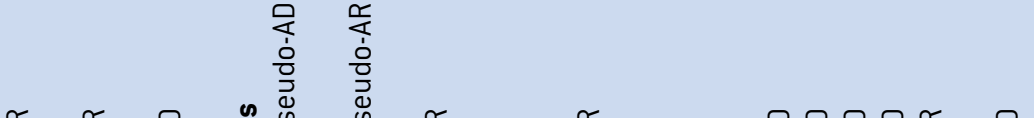

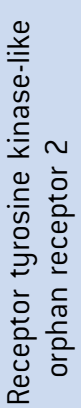

$\frac{5}{25}$

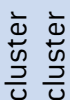

ญ ֻ

임오 우

.

든 등

을 음

윽 윽

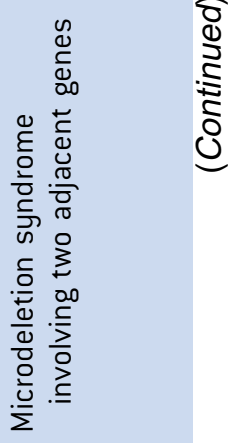

峁 的

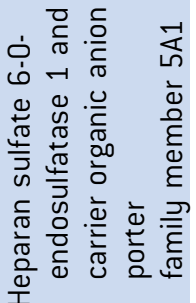

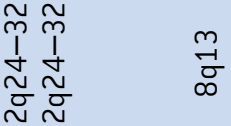

是是是是采是

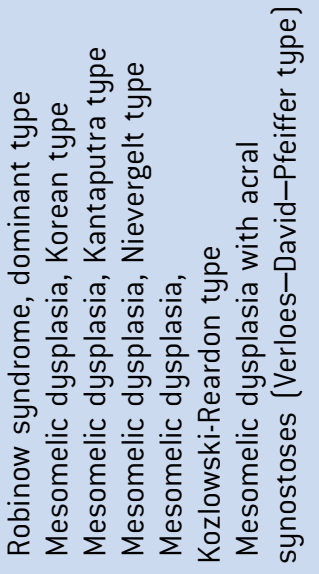




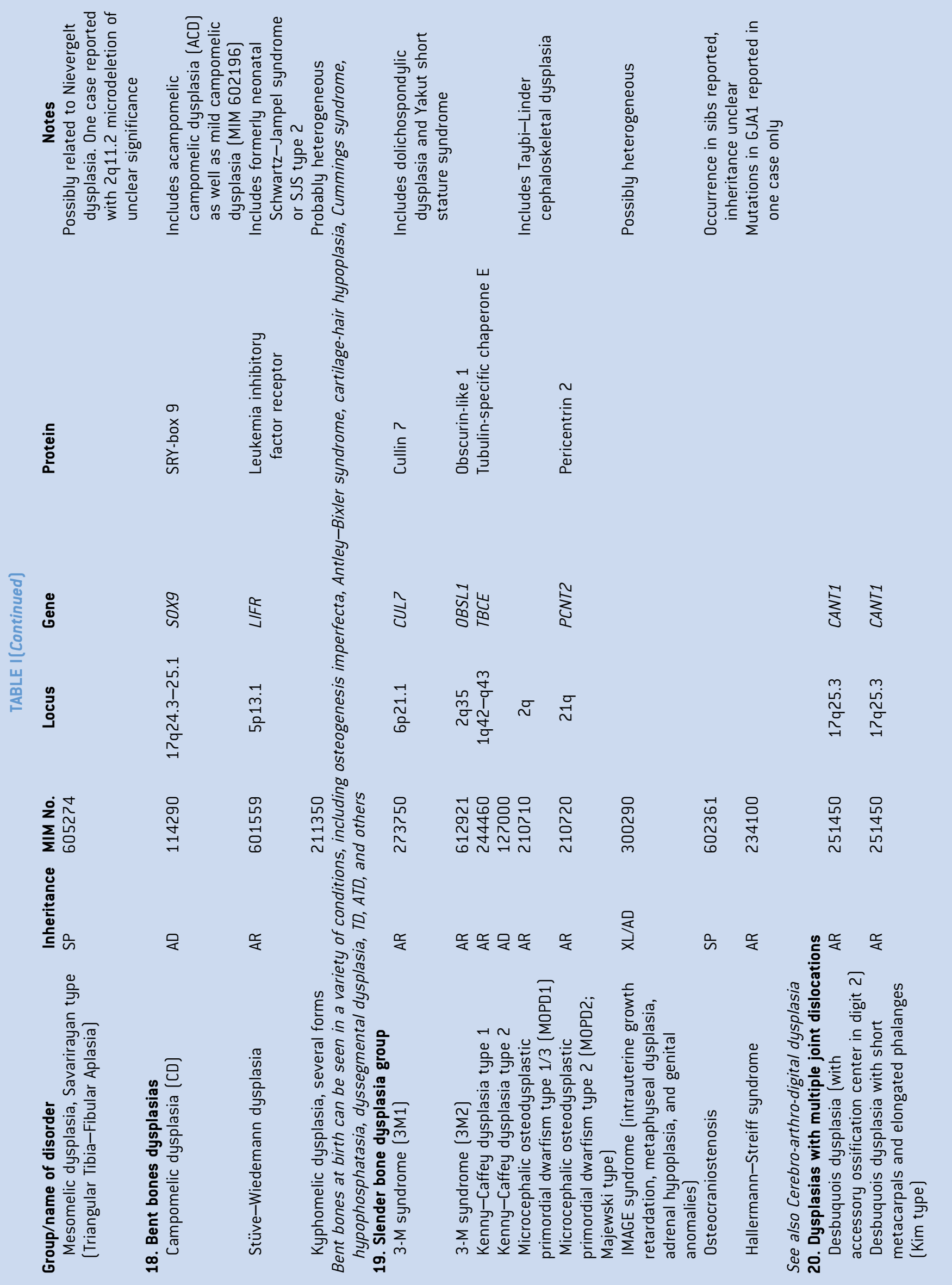




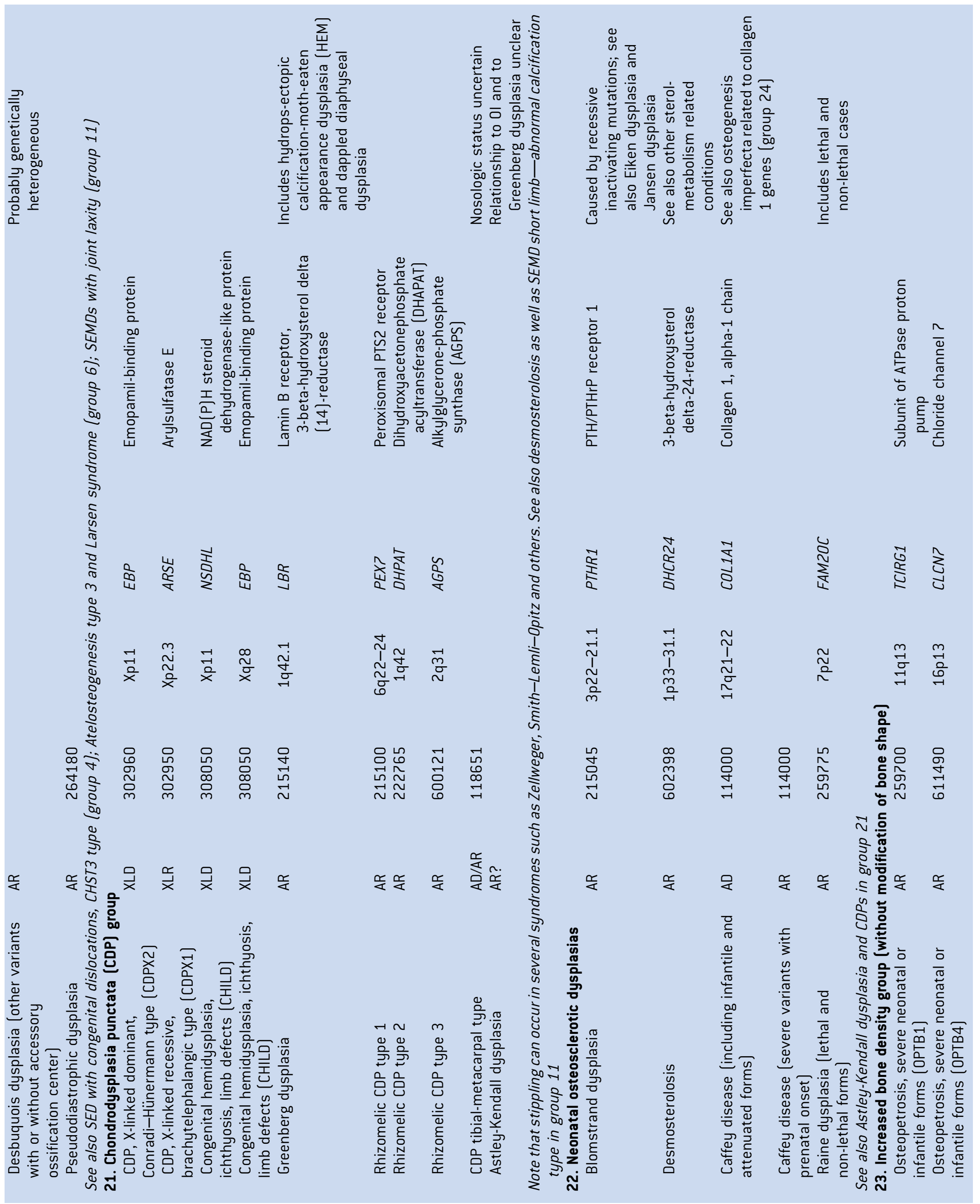



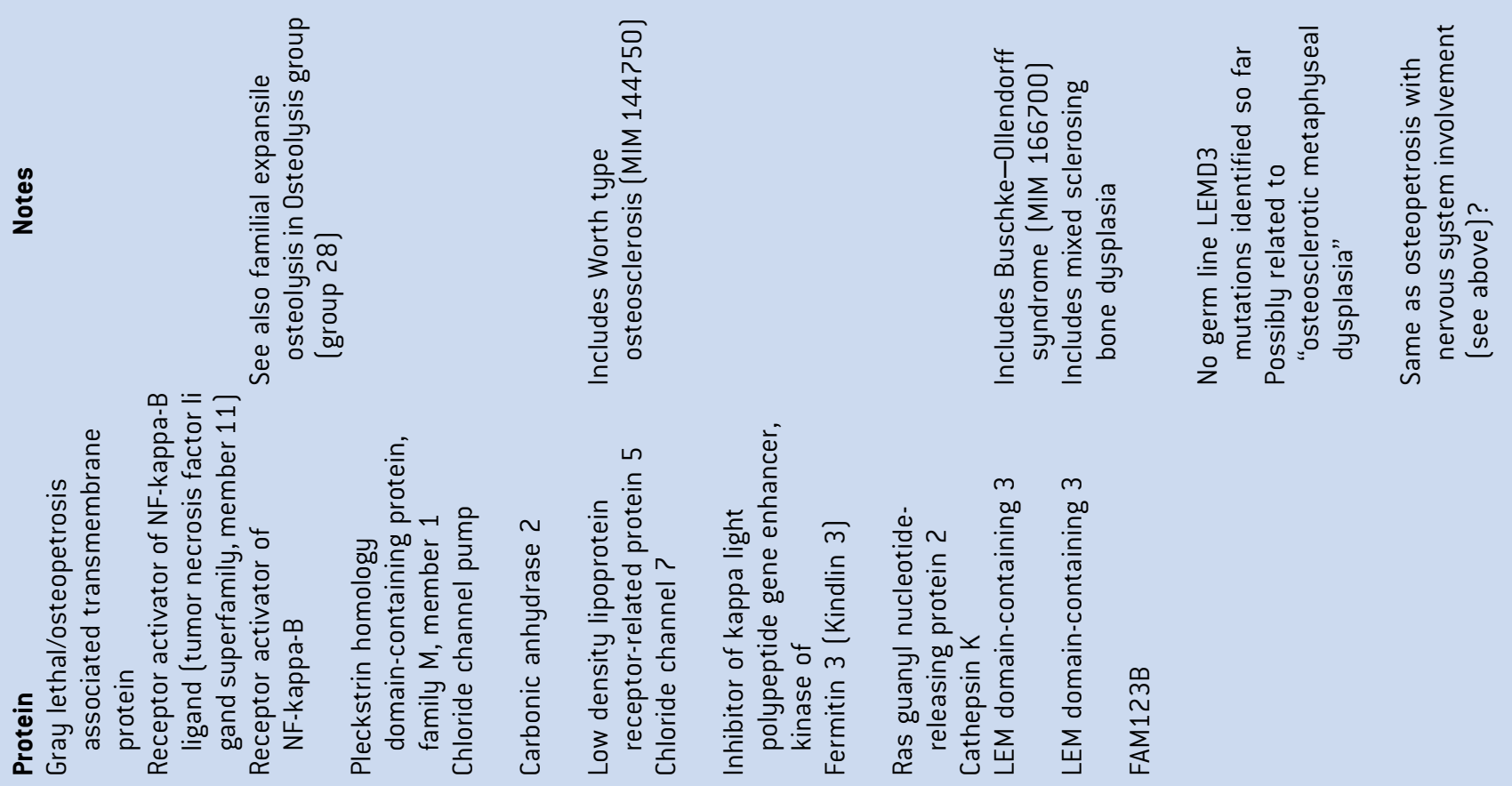

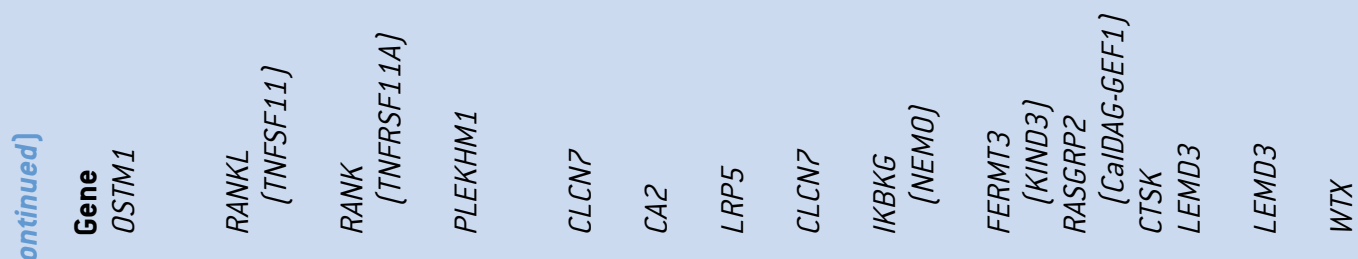

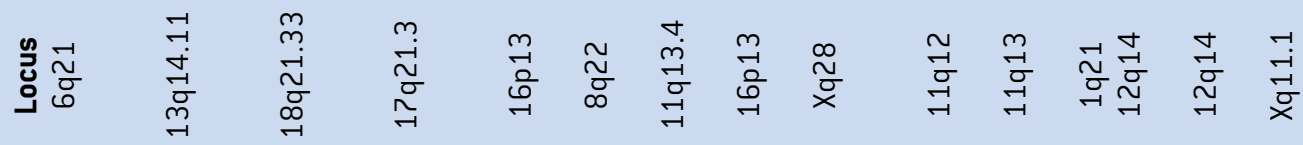

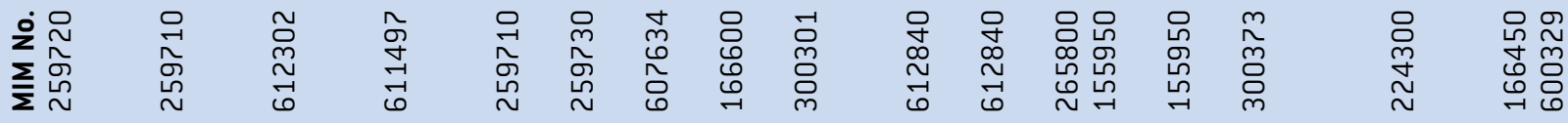

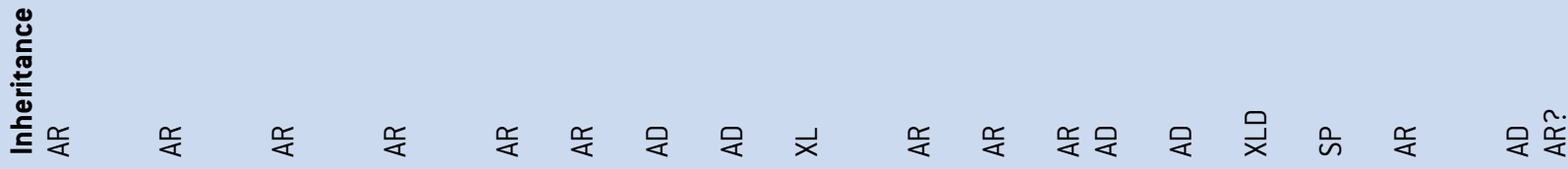

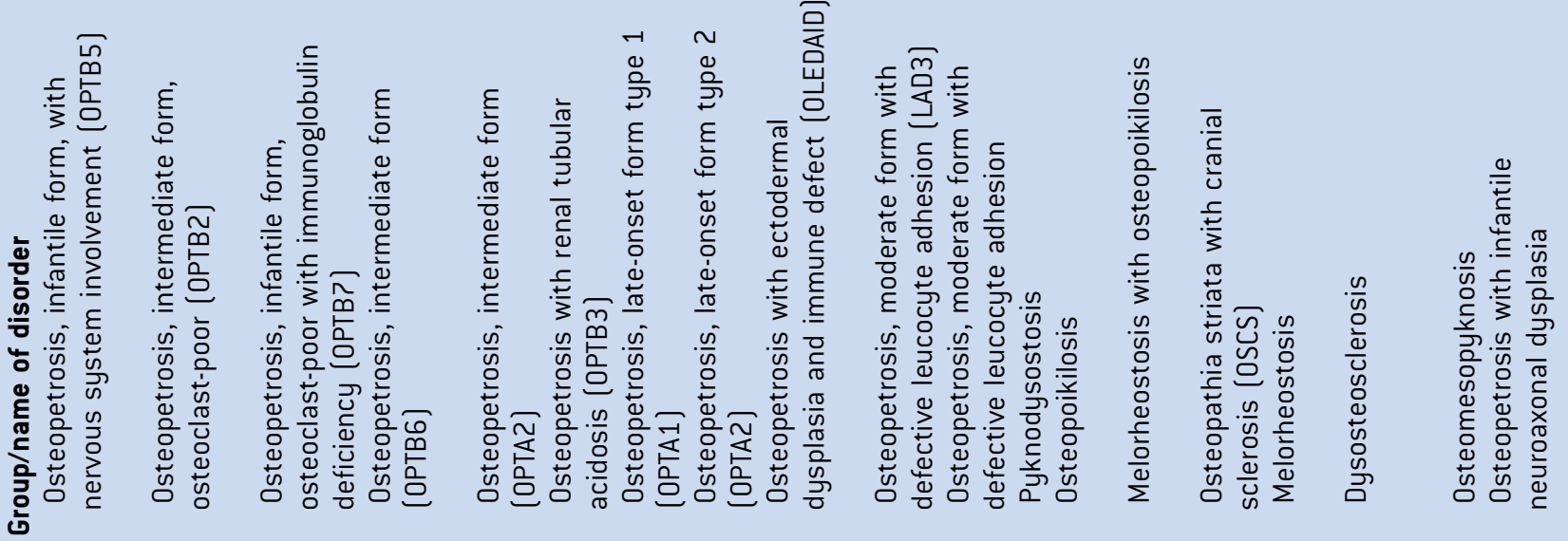



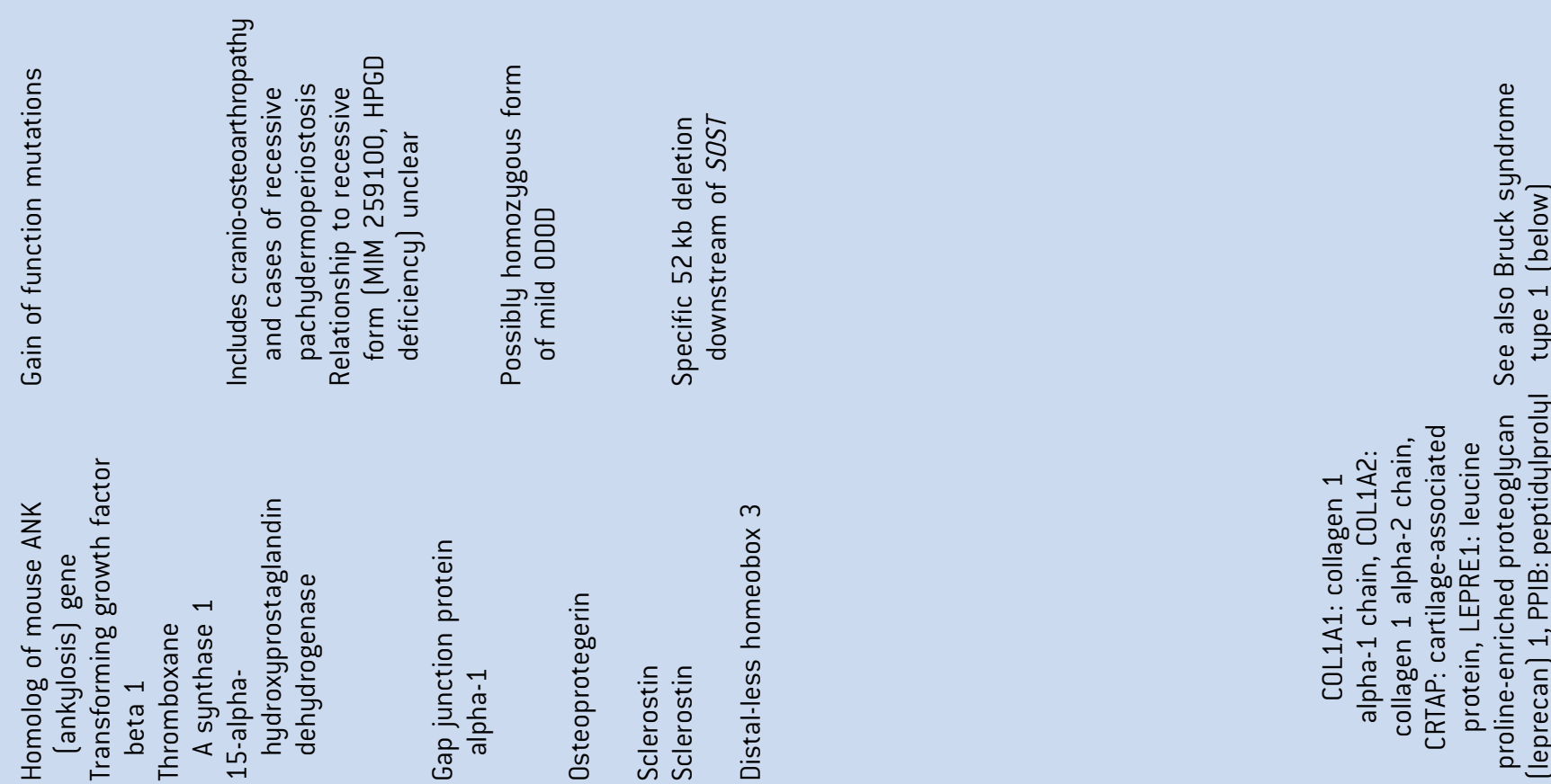

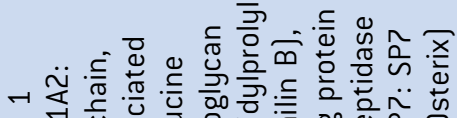

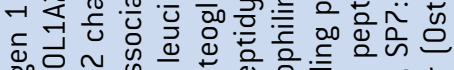

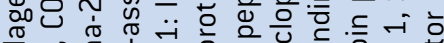

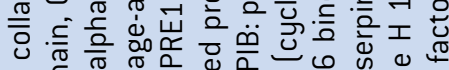

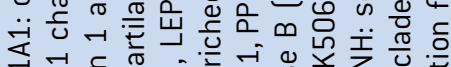

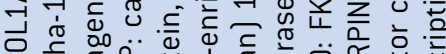
응 $\frac{\bar{c}}{\sigma}$

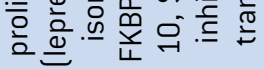

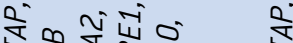

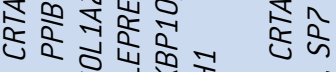

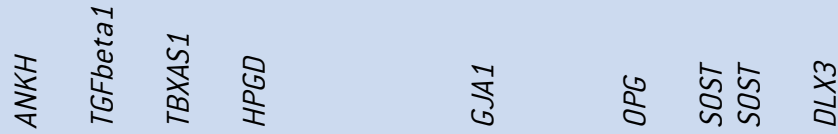

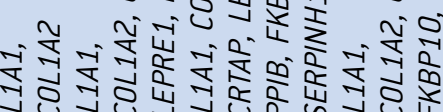

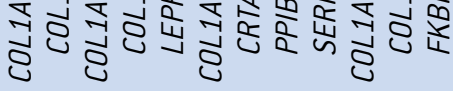

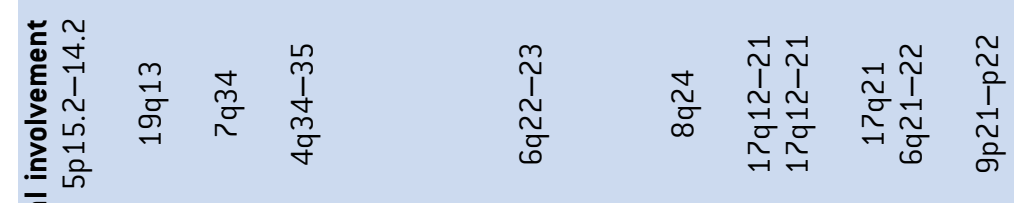

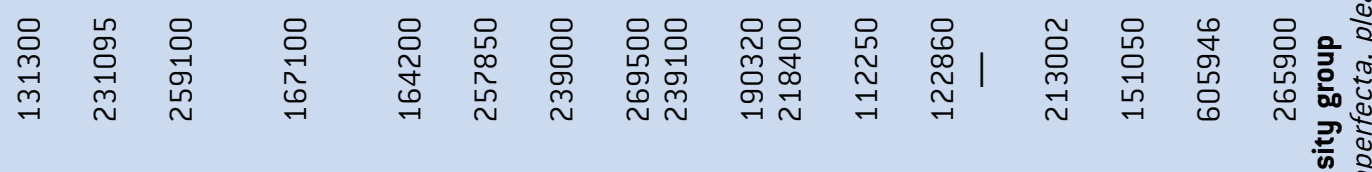
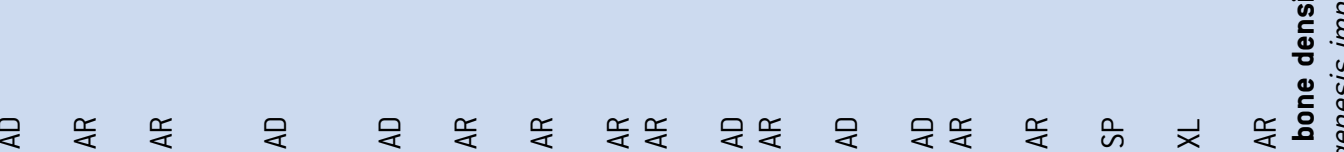

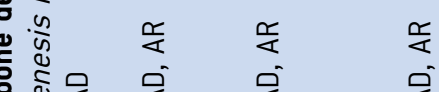



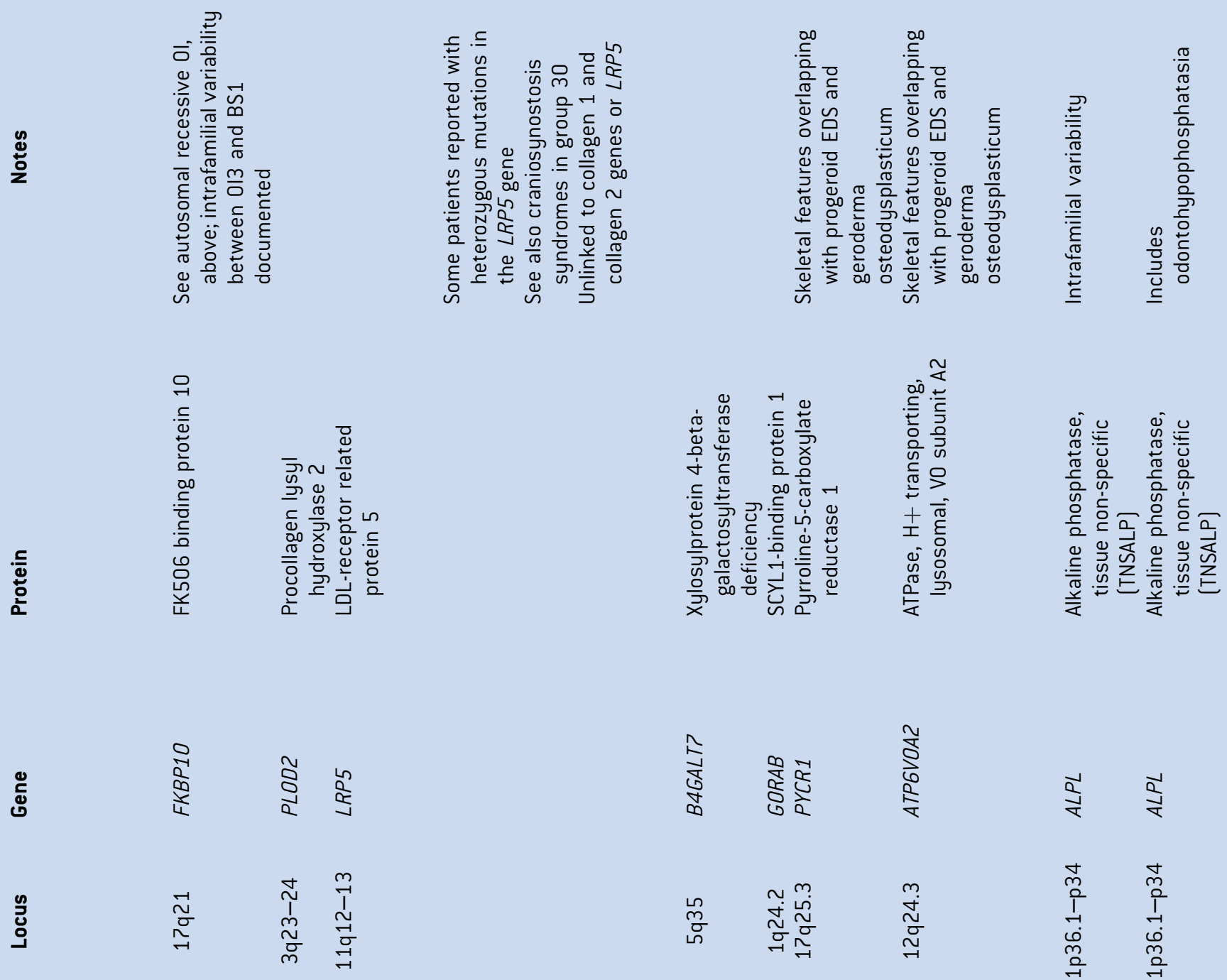

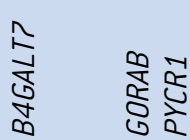

$\frac{1}{5}$
$\frac{1}{6}$
$\frac{1}{x}$

$\frac{\pi}{\frac{\pi}{4}}$

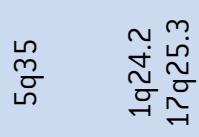

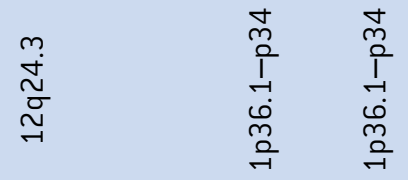

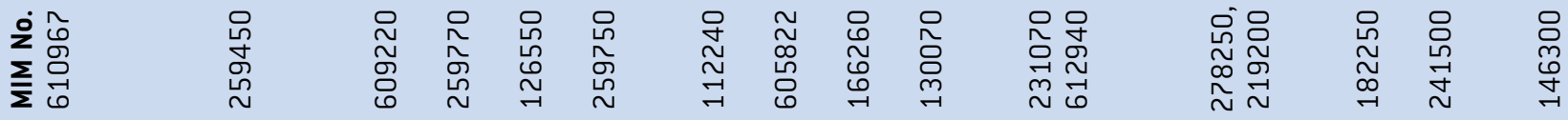

冚

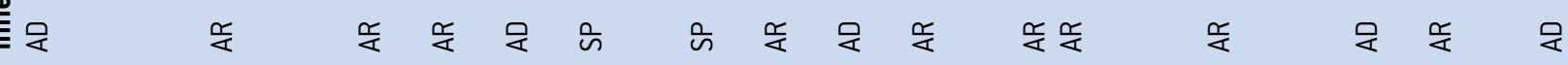

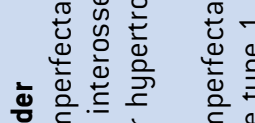

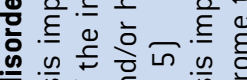

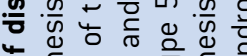

割

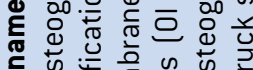

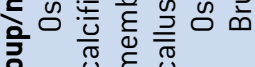
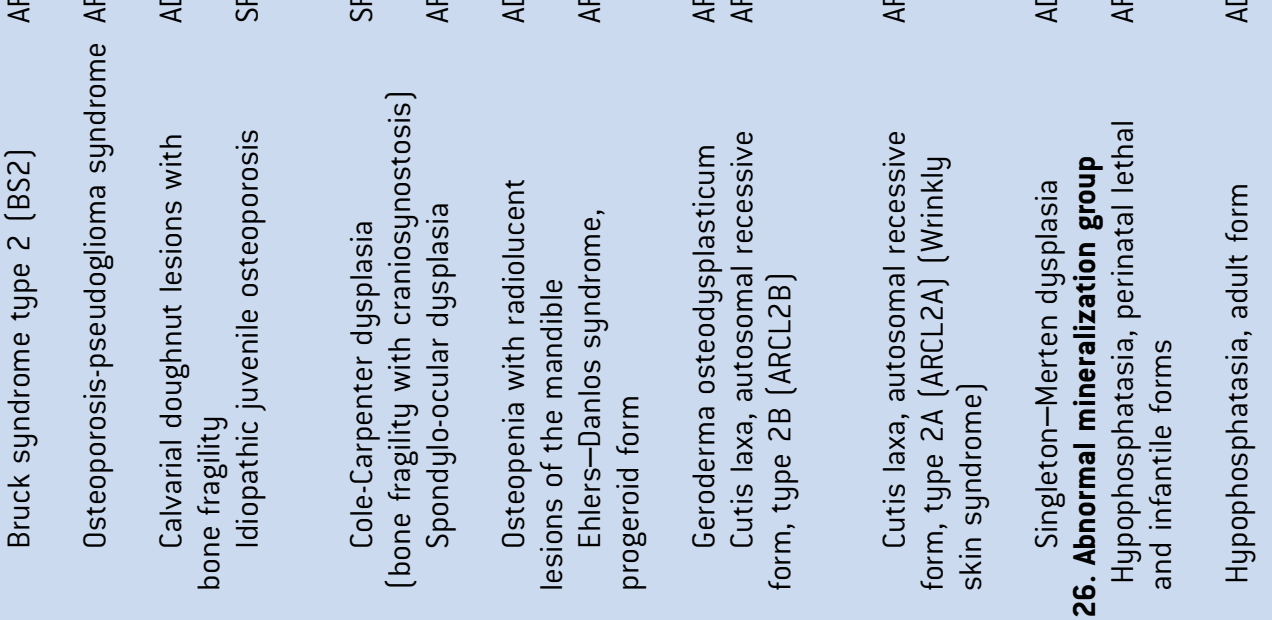


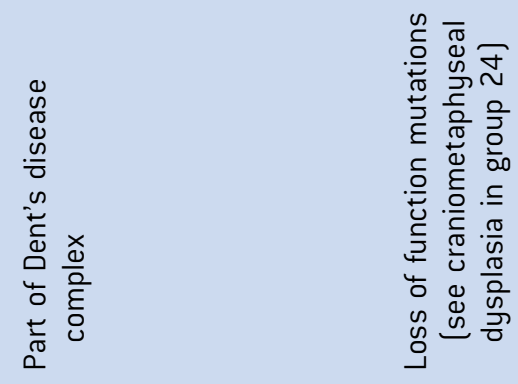

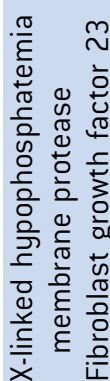
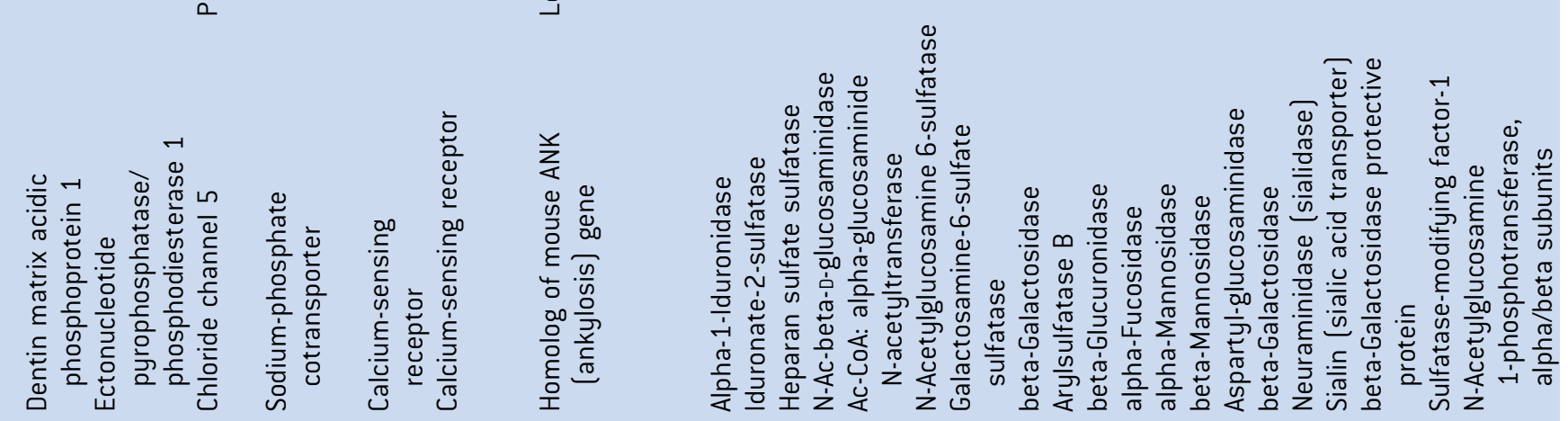

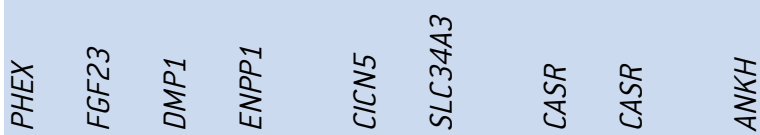

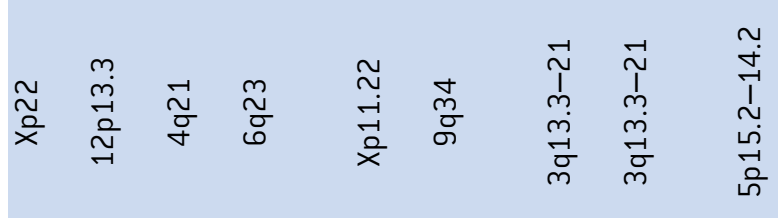

\section{高}

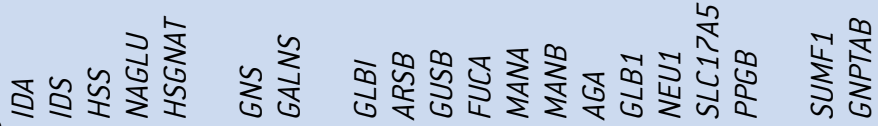

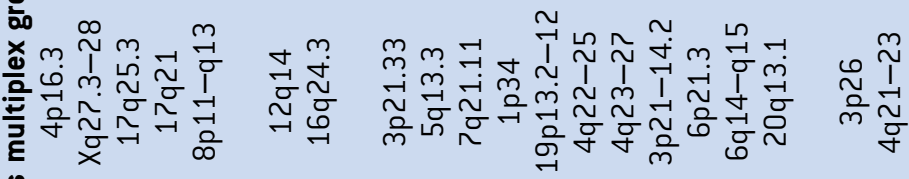

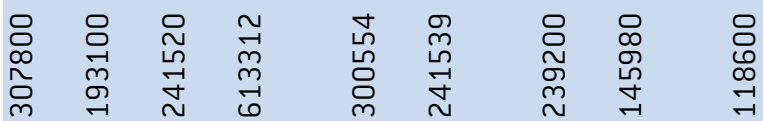

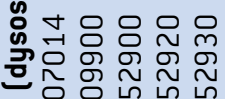

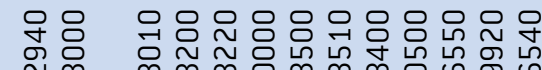
N

옹유

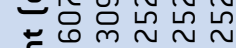

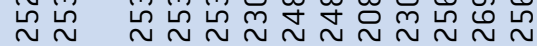

$\approx \widetilde{\sim}$

\section{突}

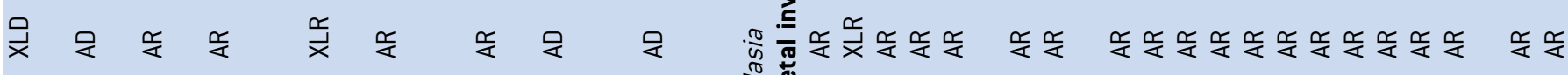

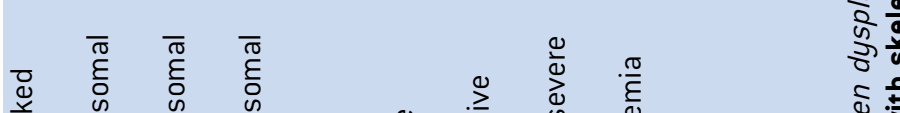

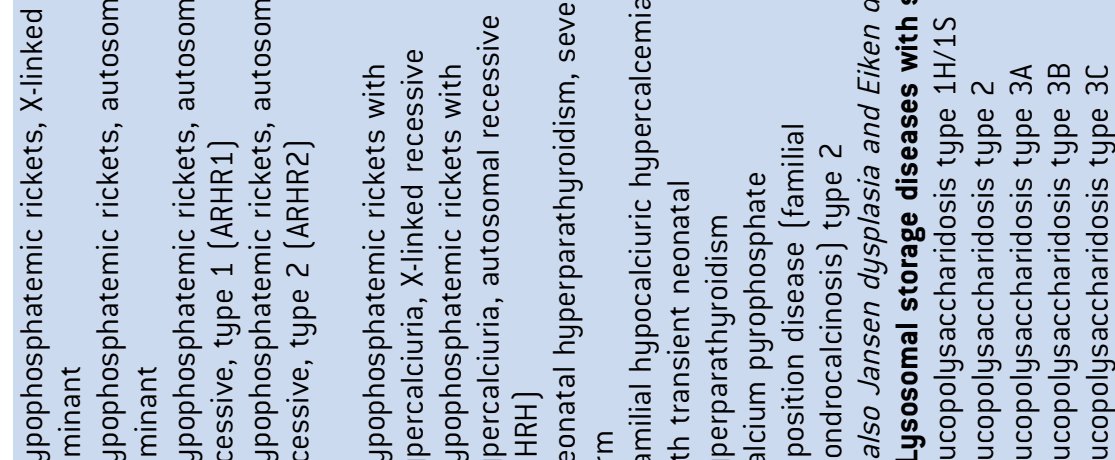

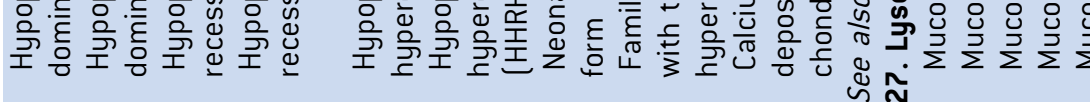

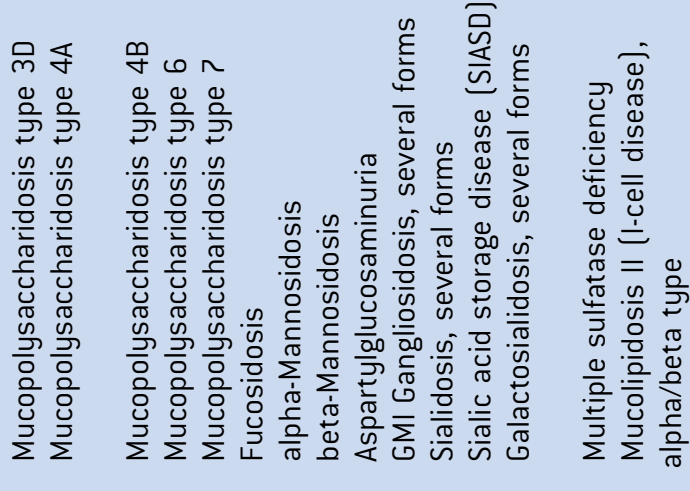



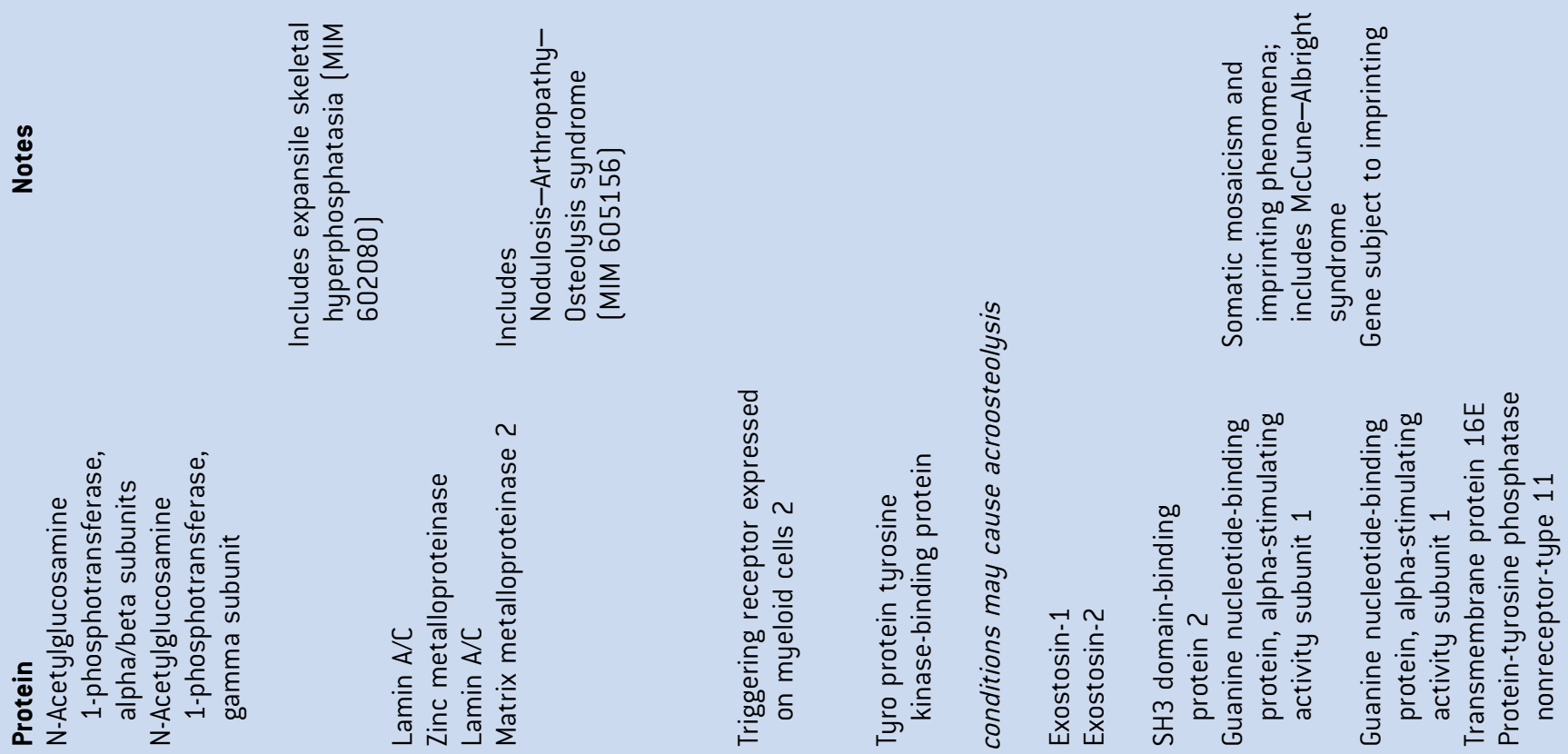

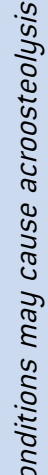

-

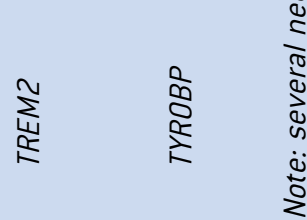

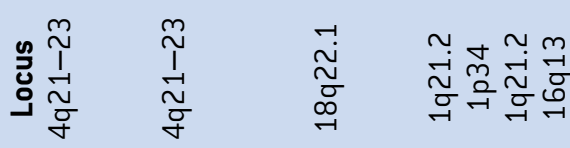

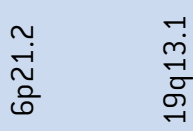

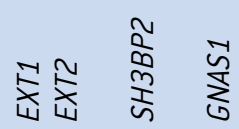

$\sum_{\substack{\infty \\ \sum}}^{\substack{\infty \\ \sum}}$ $\stackrel{i}{i}$

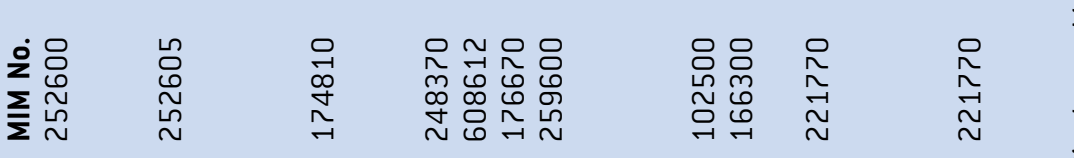

巡

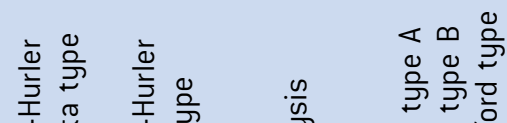

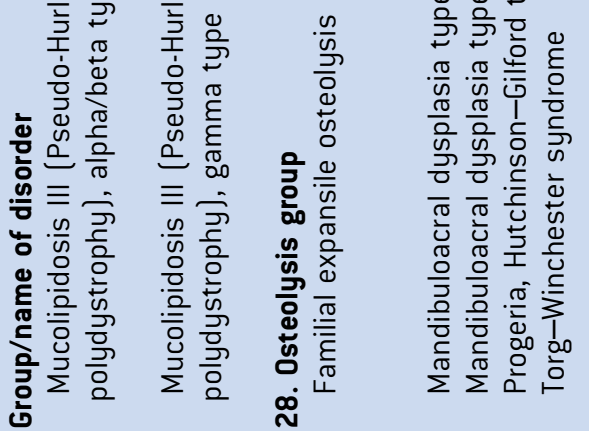

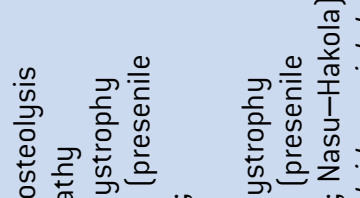

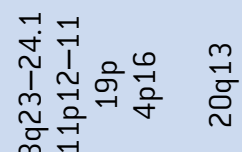

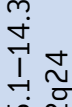

$\infty \neg$

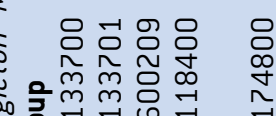

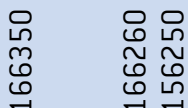

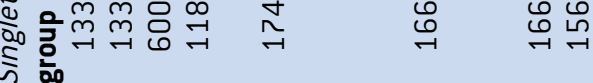

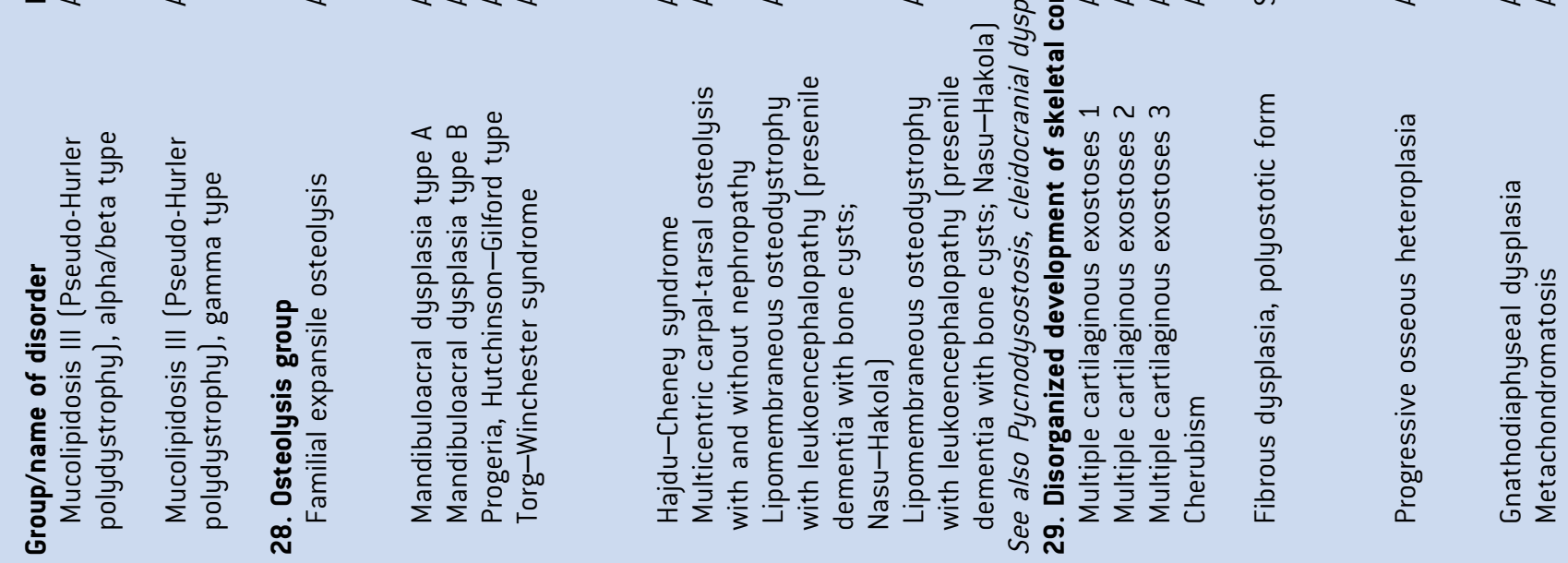



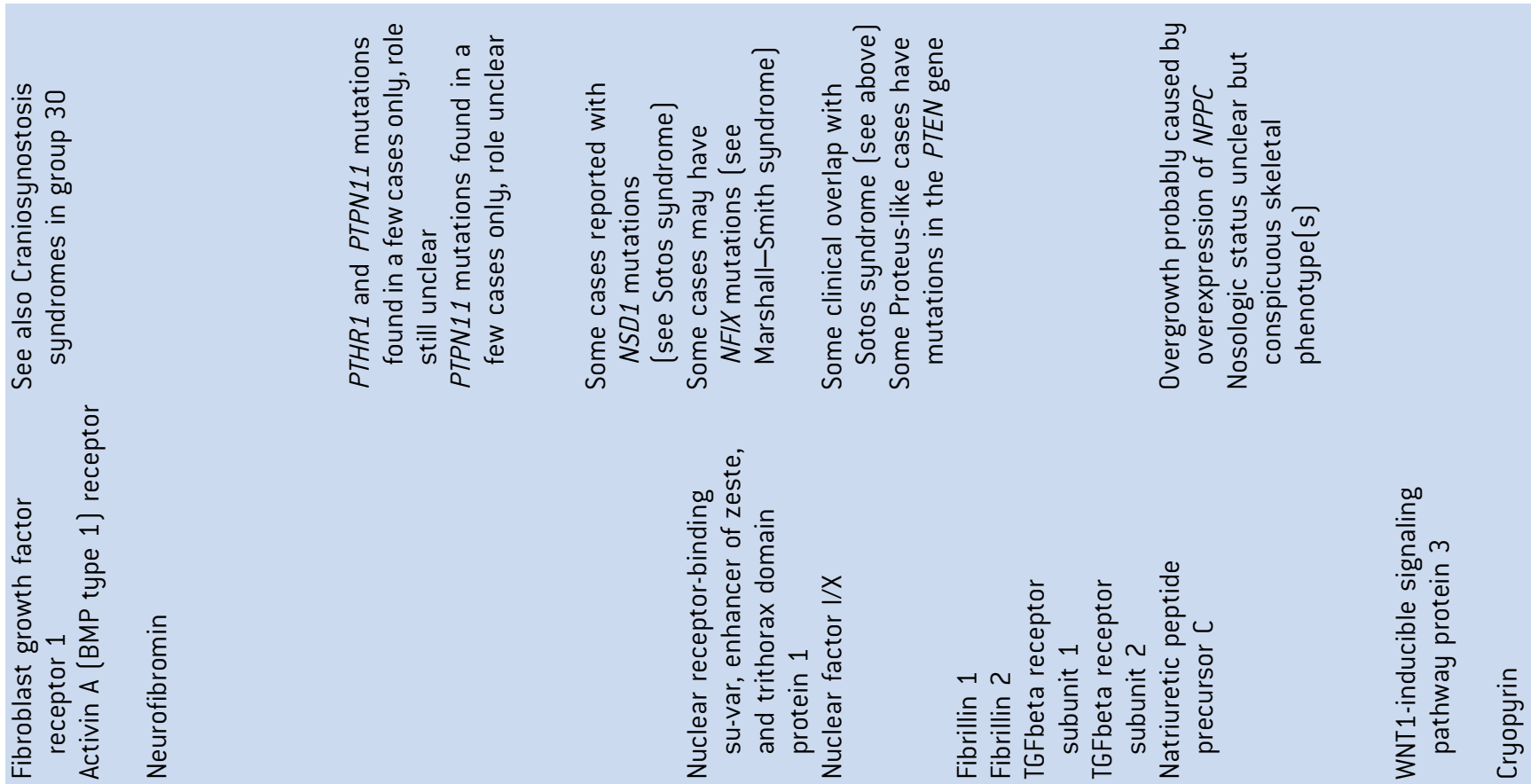

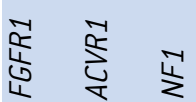

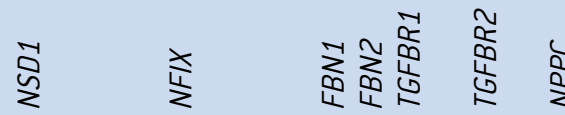

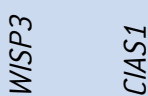

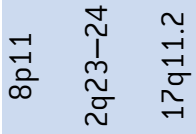

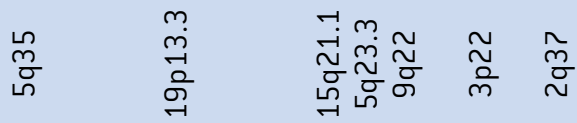

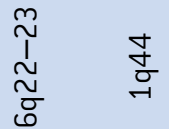

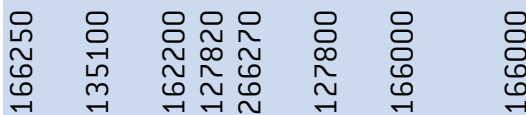

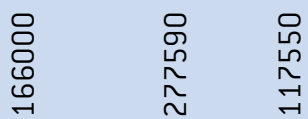

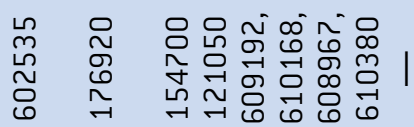

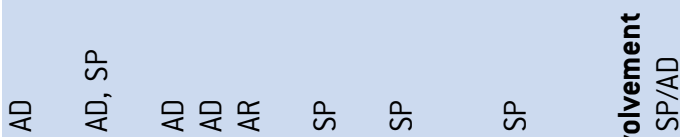

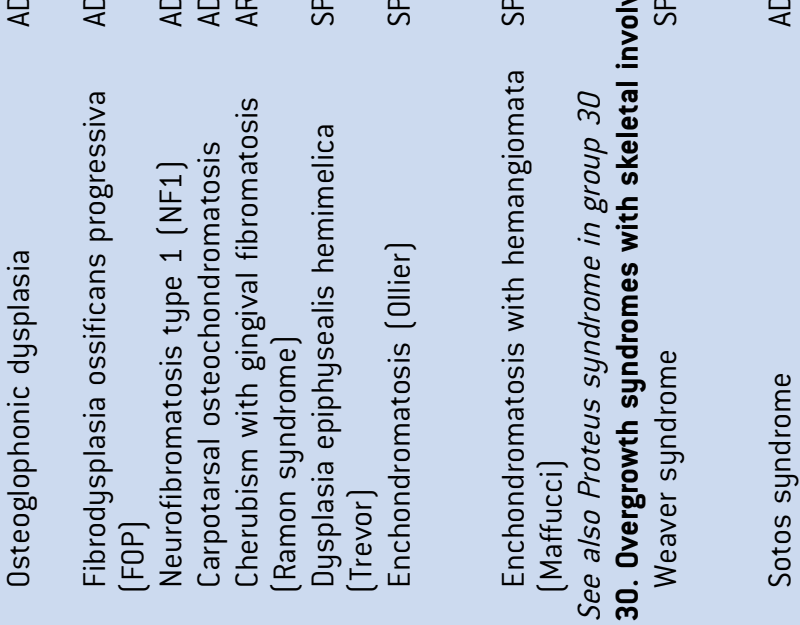

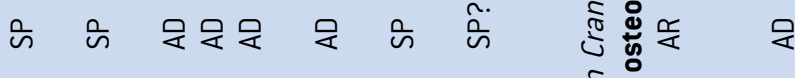

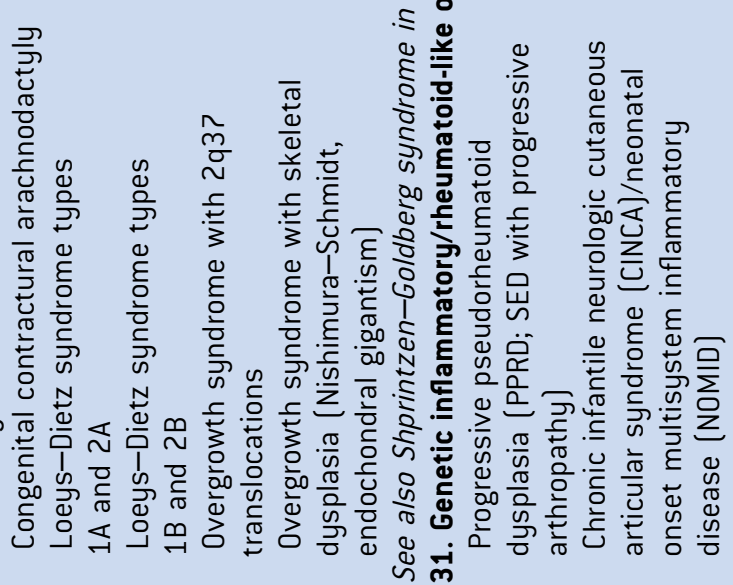



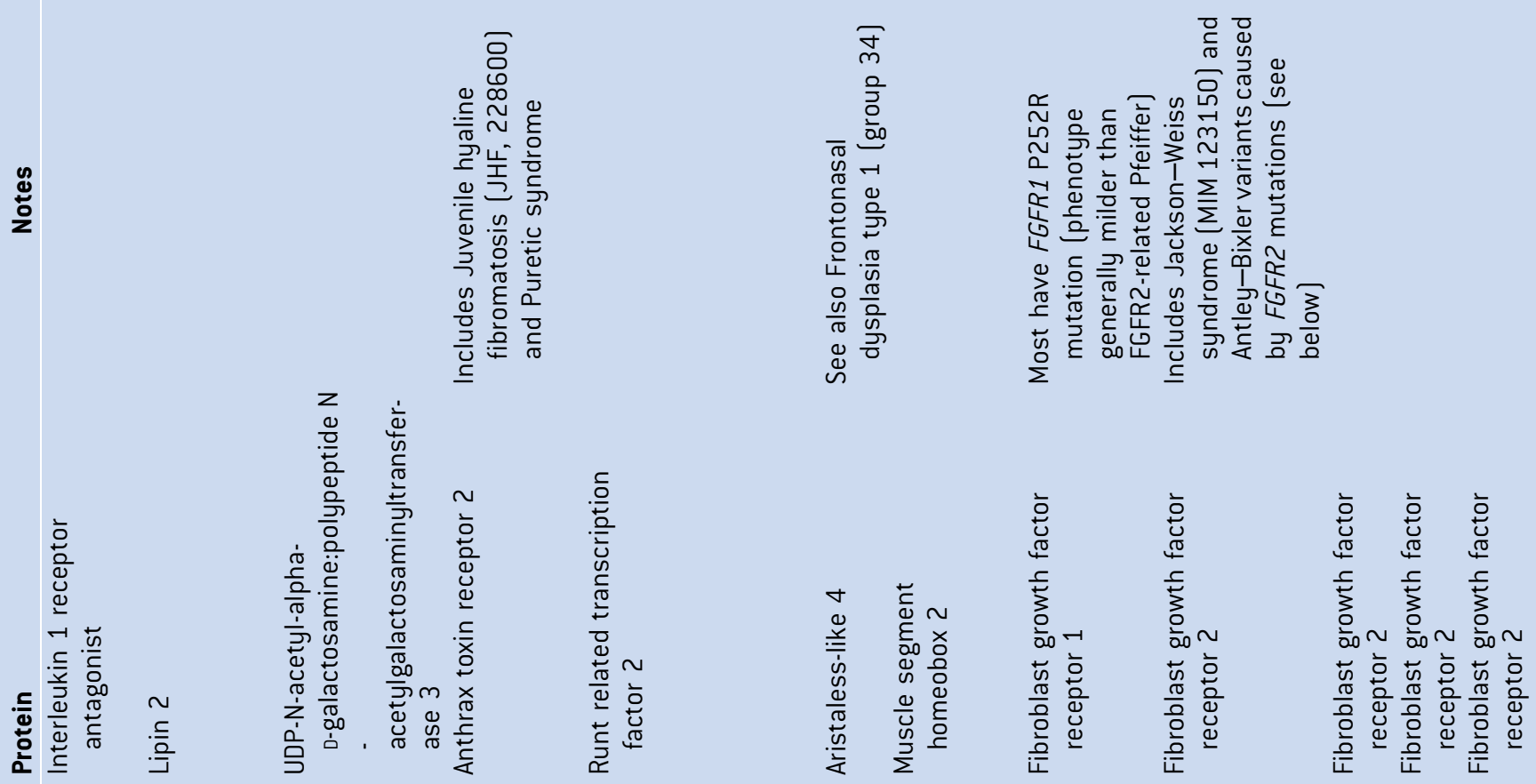

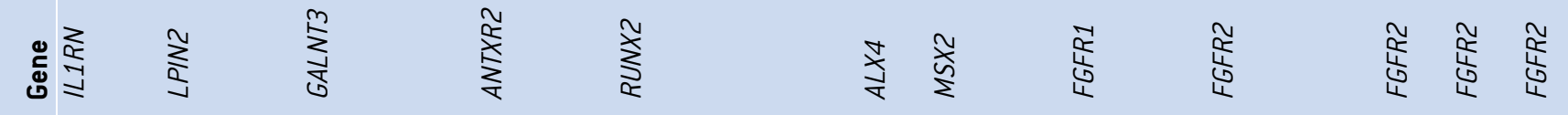

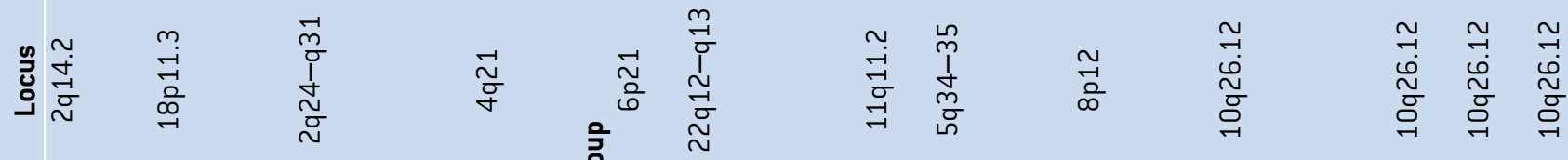

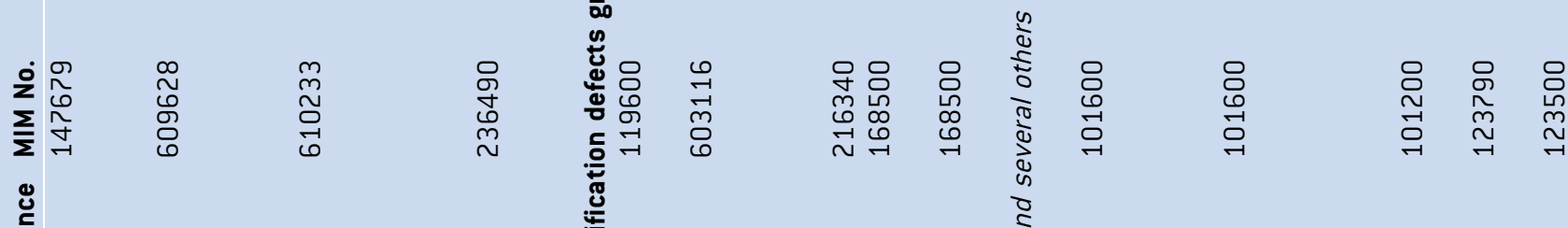

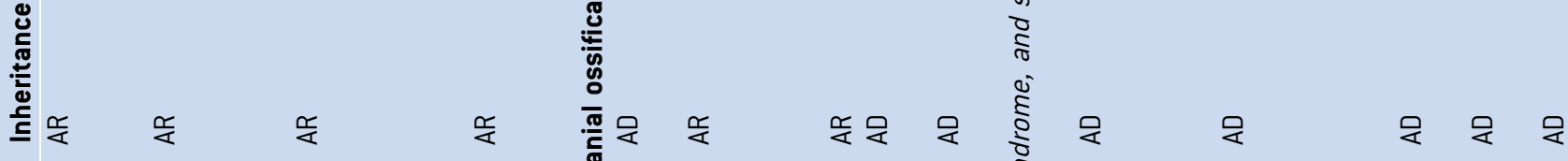

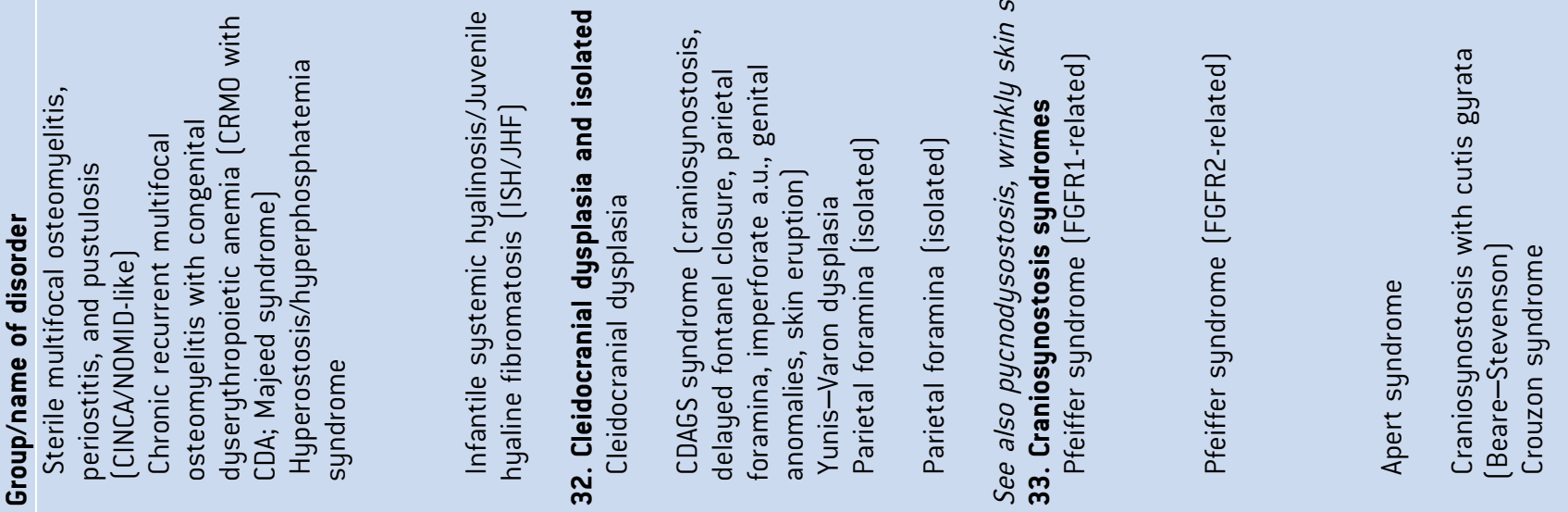




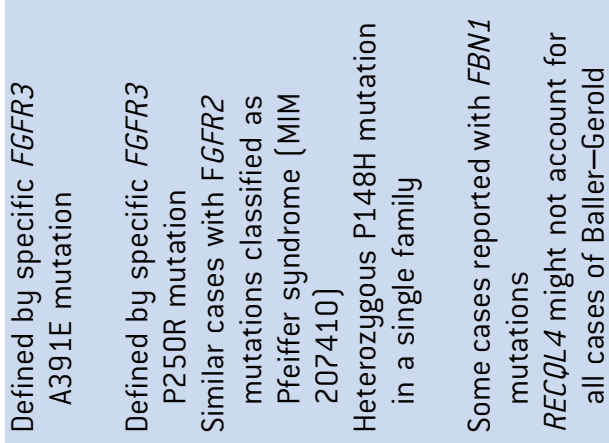

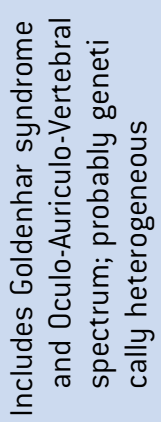

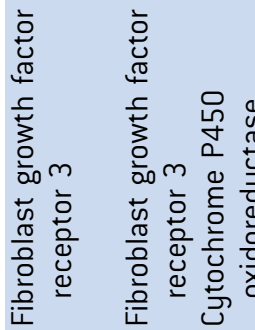

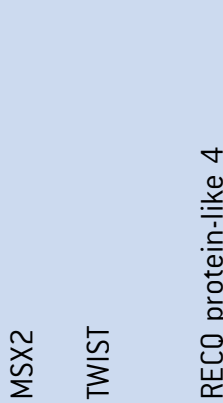

+े

E⿱士

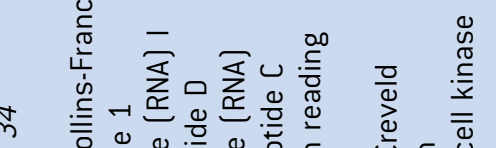

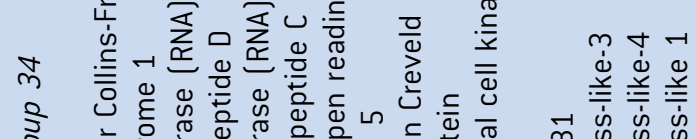

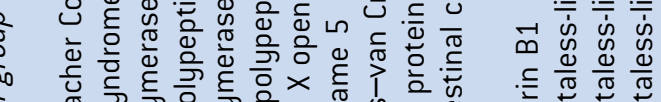

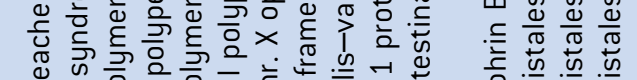

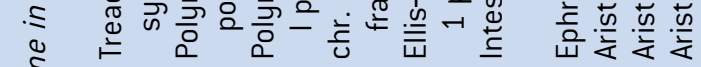

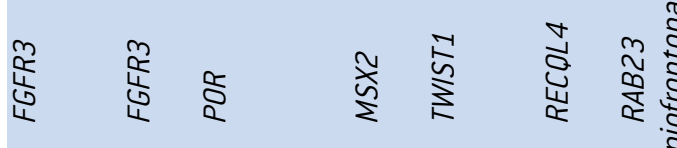

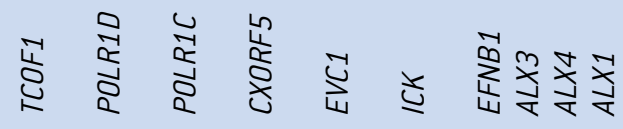

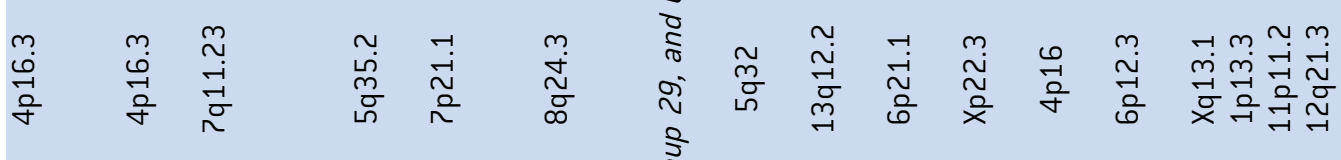

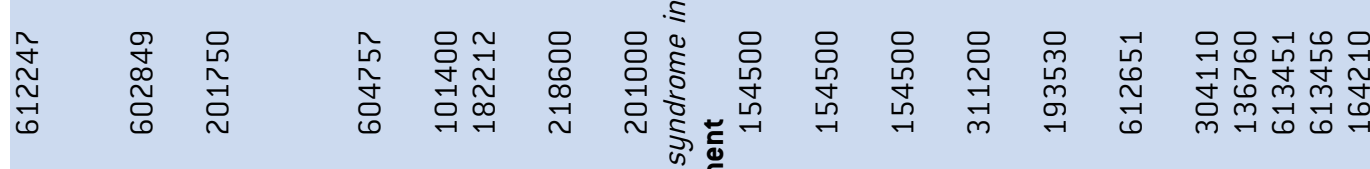

究

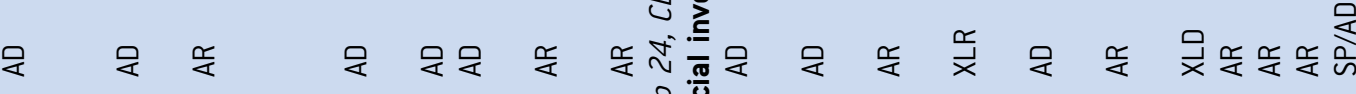

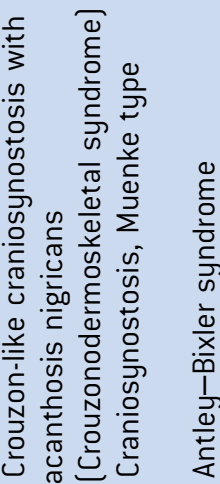

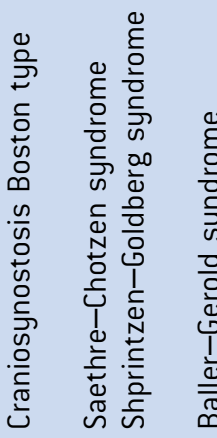

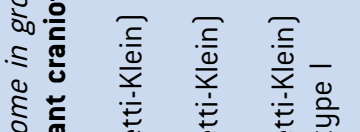

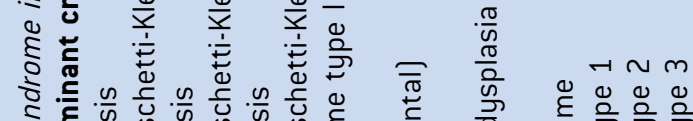
w

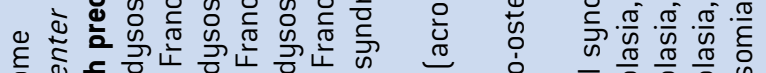
क⿺辶一兀

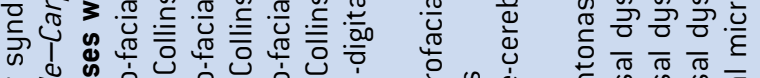

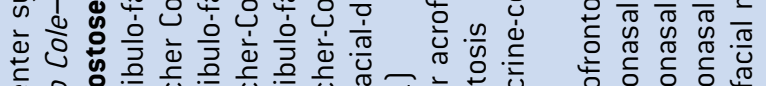

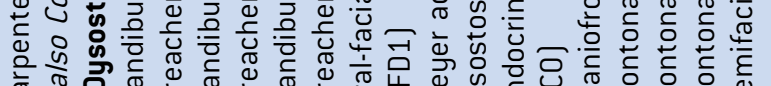

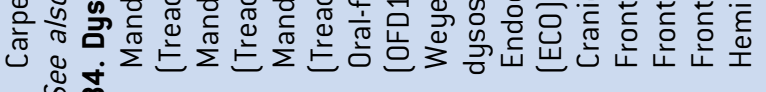

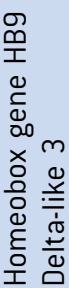

ำ

ํㅗㄴ

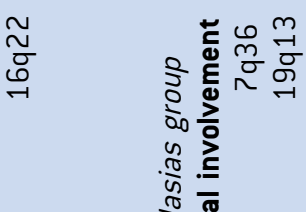

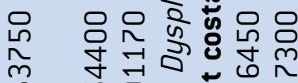

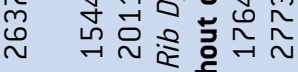

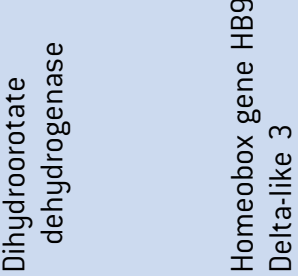

ธิ

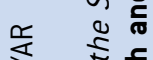

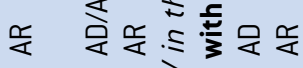

\&

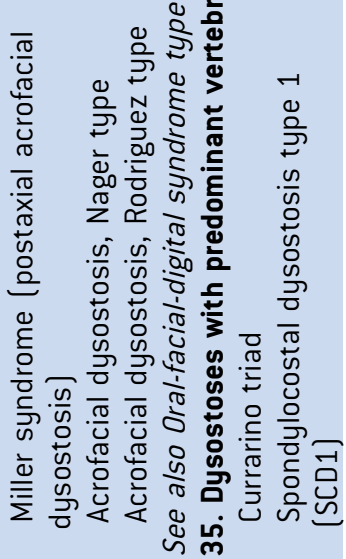




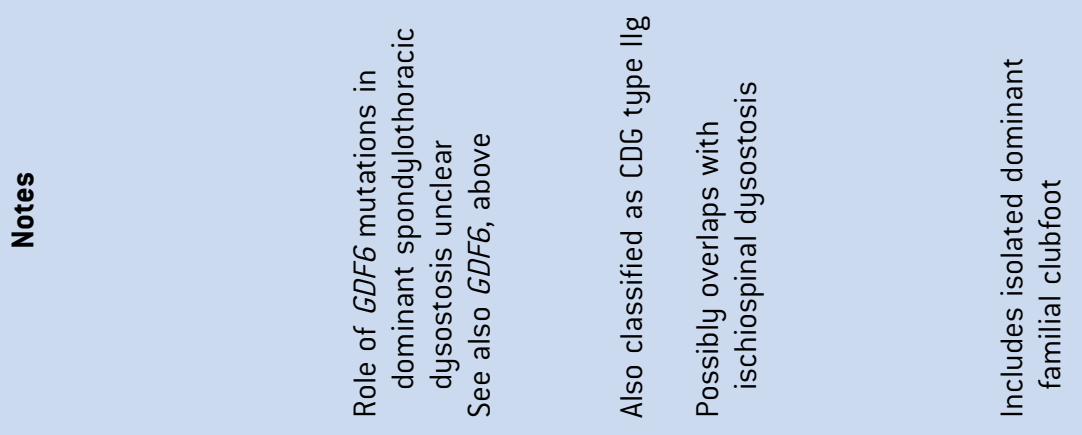

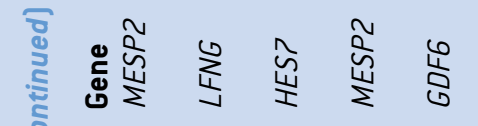
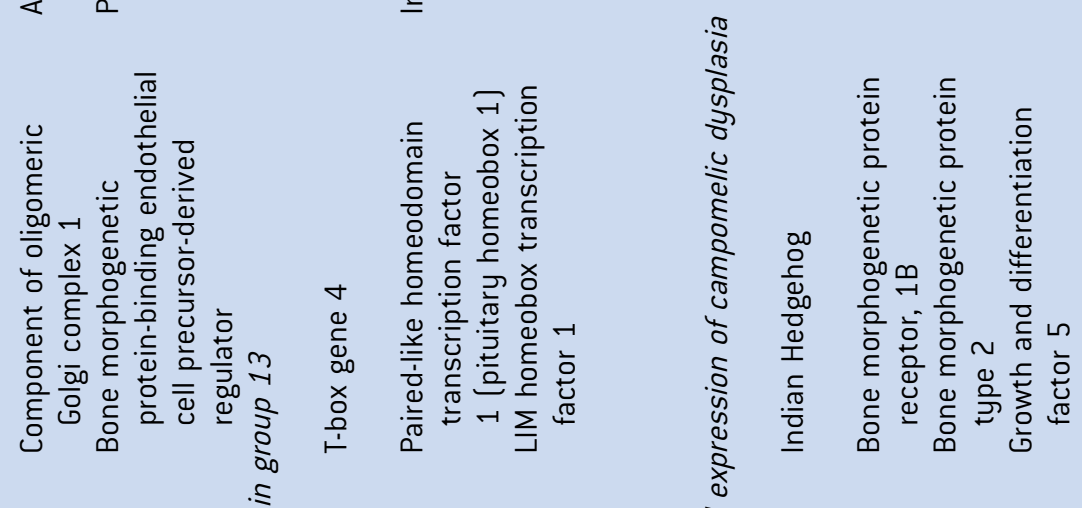

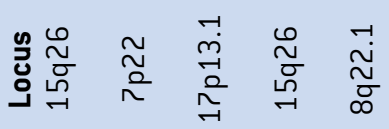

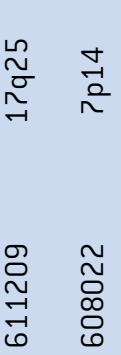

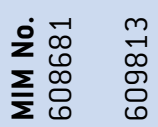

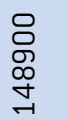

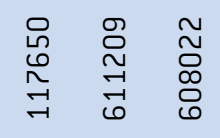

ััّ

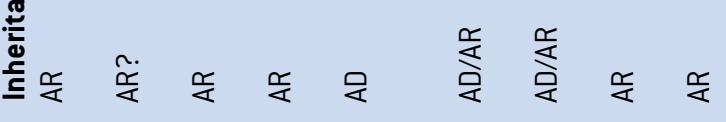

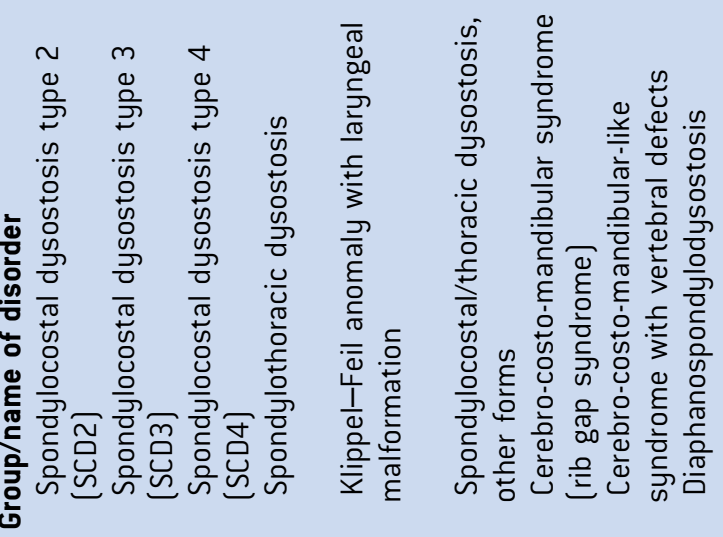

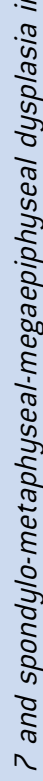



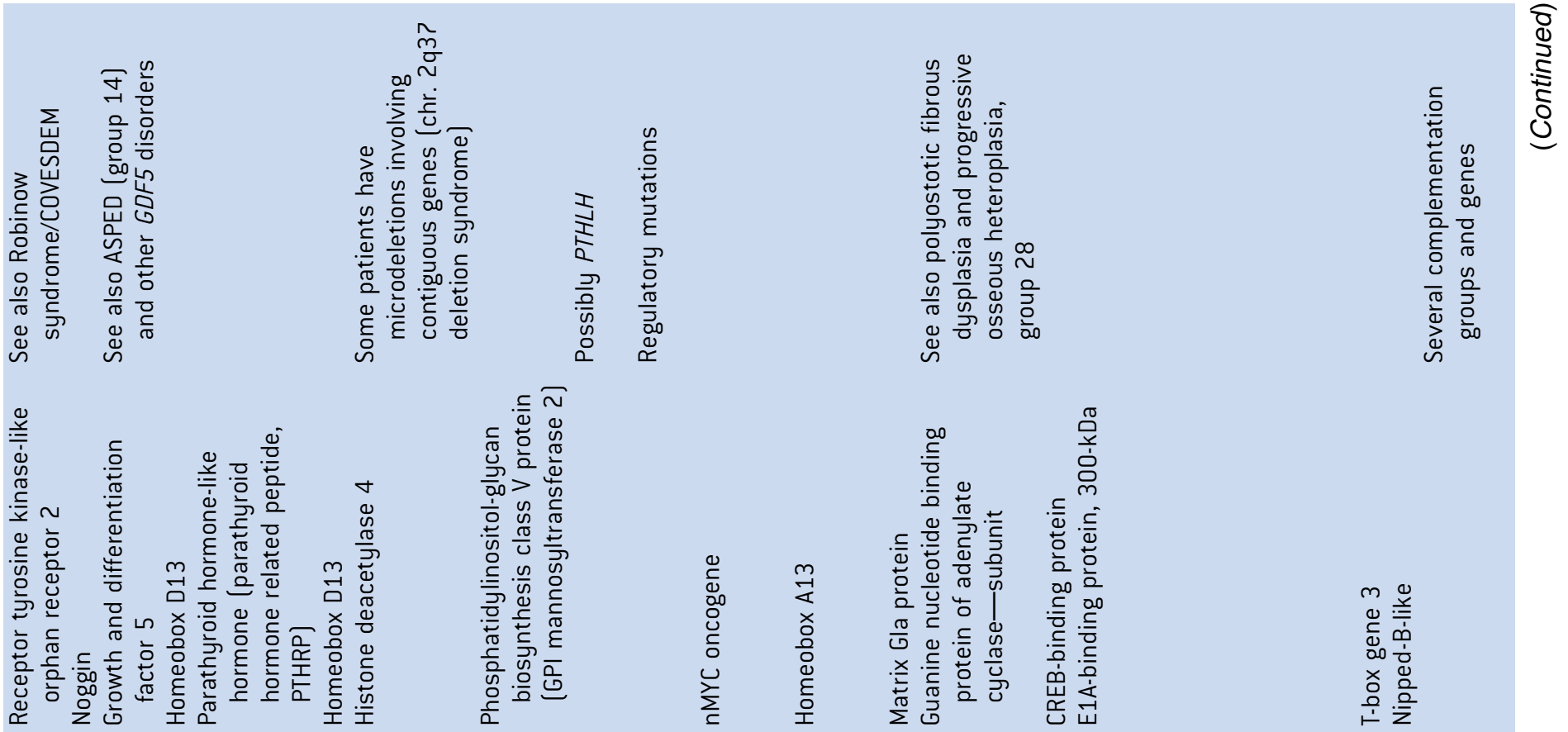

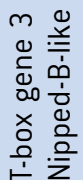

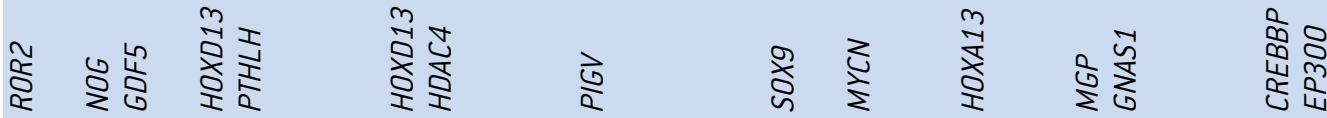
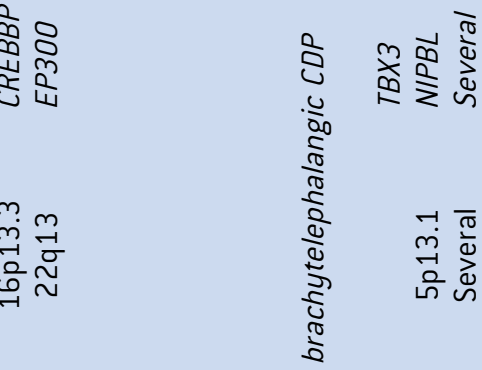

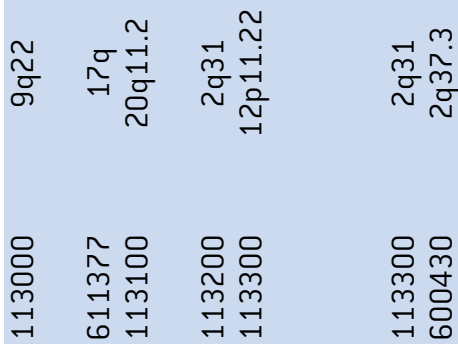

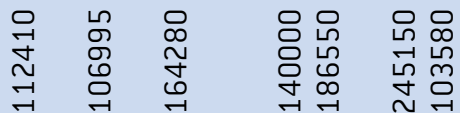

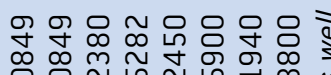

क

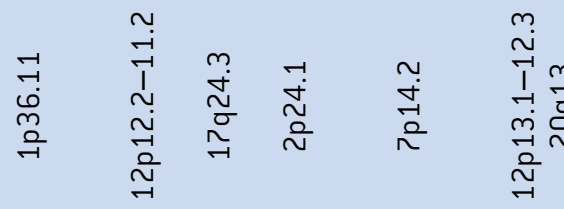

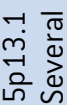

采

爱

是是安 是是

운운

学 是是是是是采是

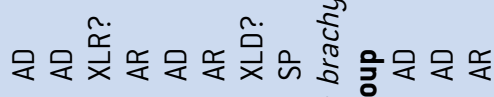

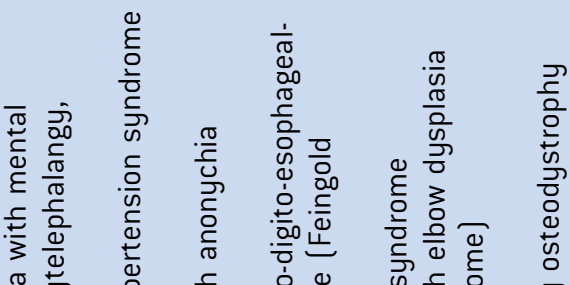

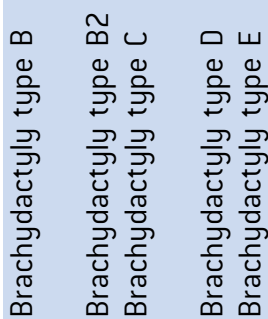

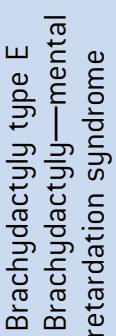

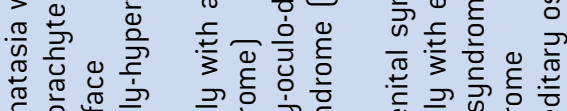

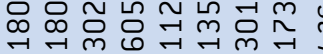

㶽号设 $\rightarrow \underset{\sim}{\sim} \approx$ $\underset{\sim}{\infty} \underset{\sim}{\sim} \approx$ 


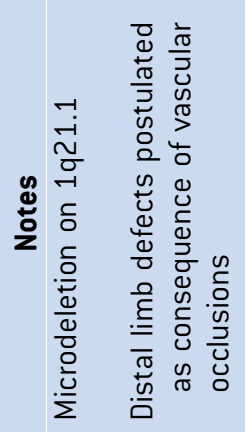

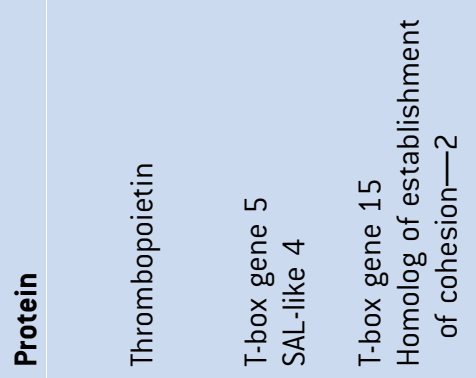
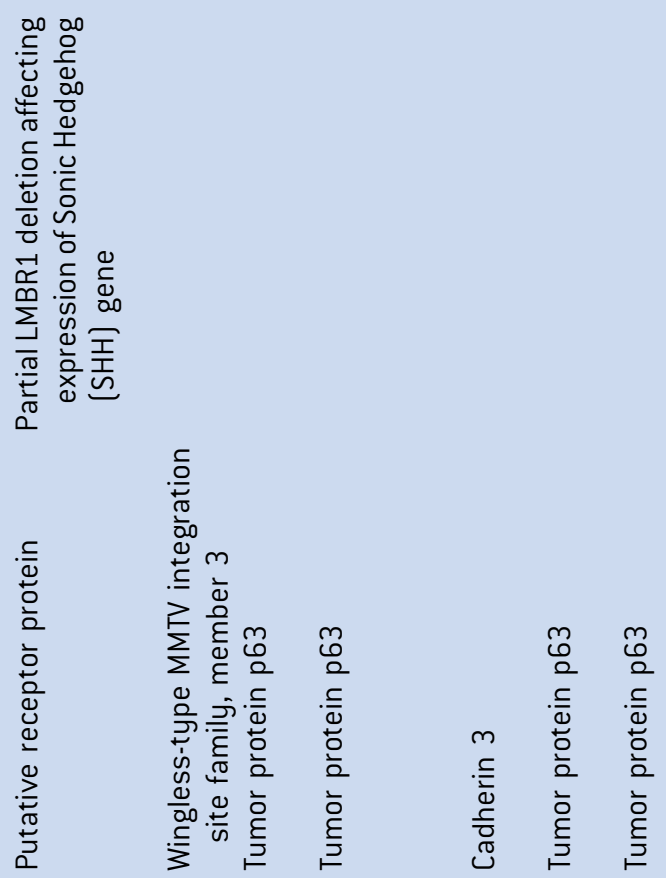

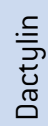

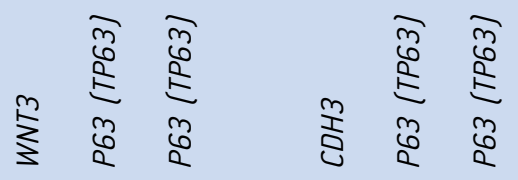

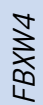

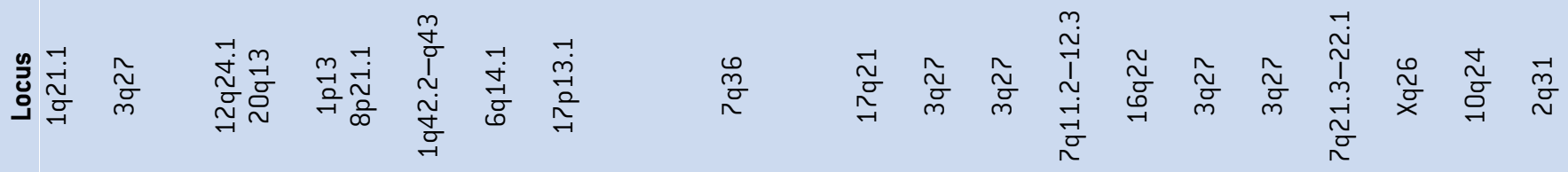

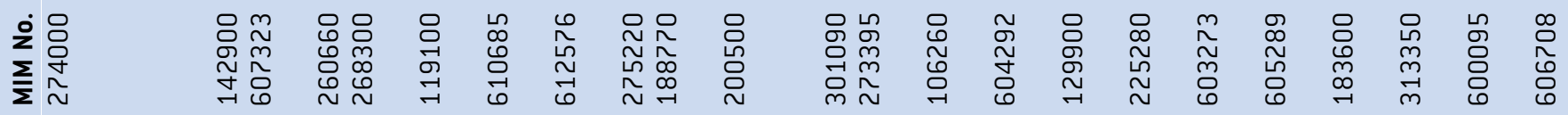
岂

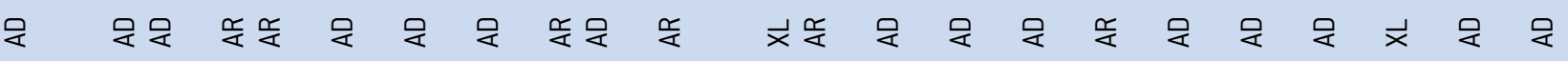
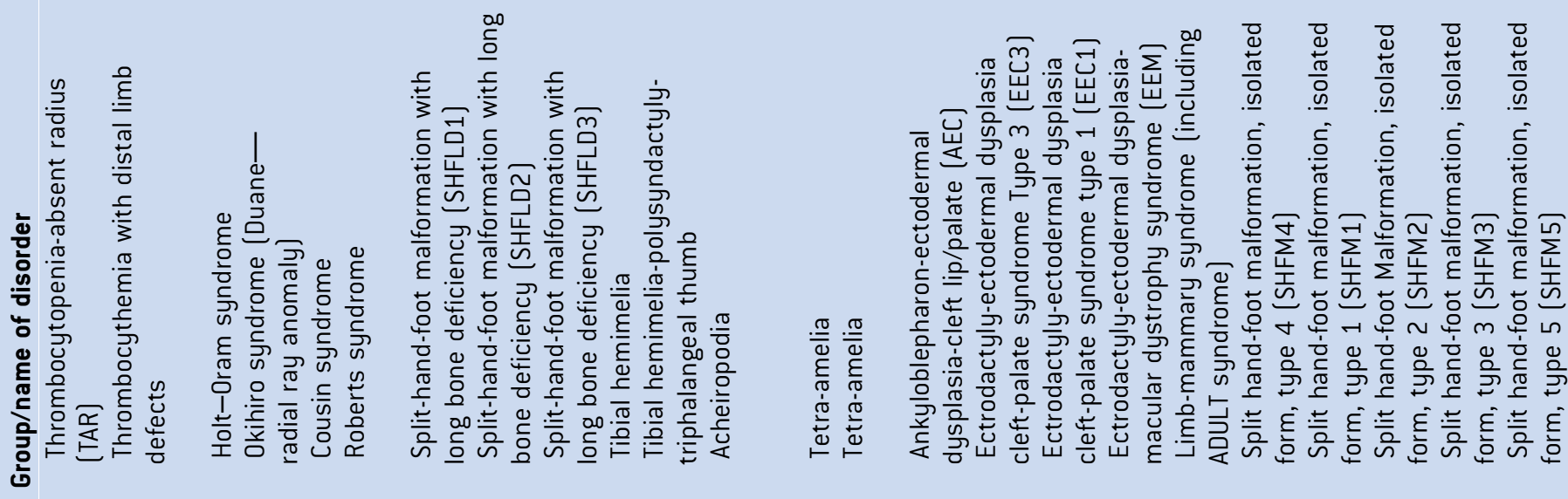


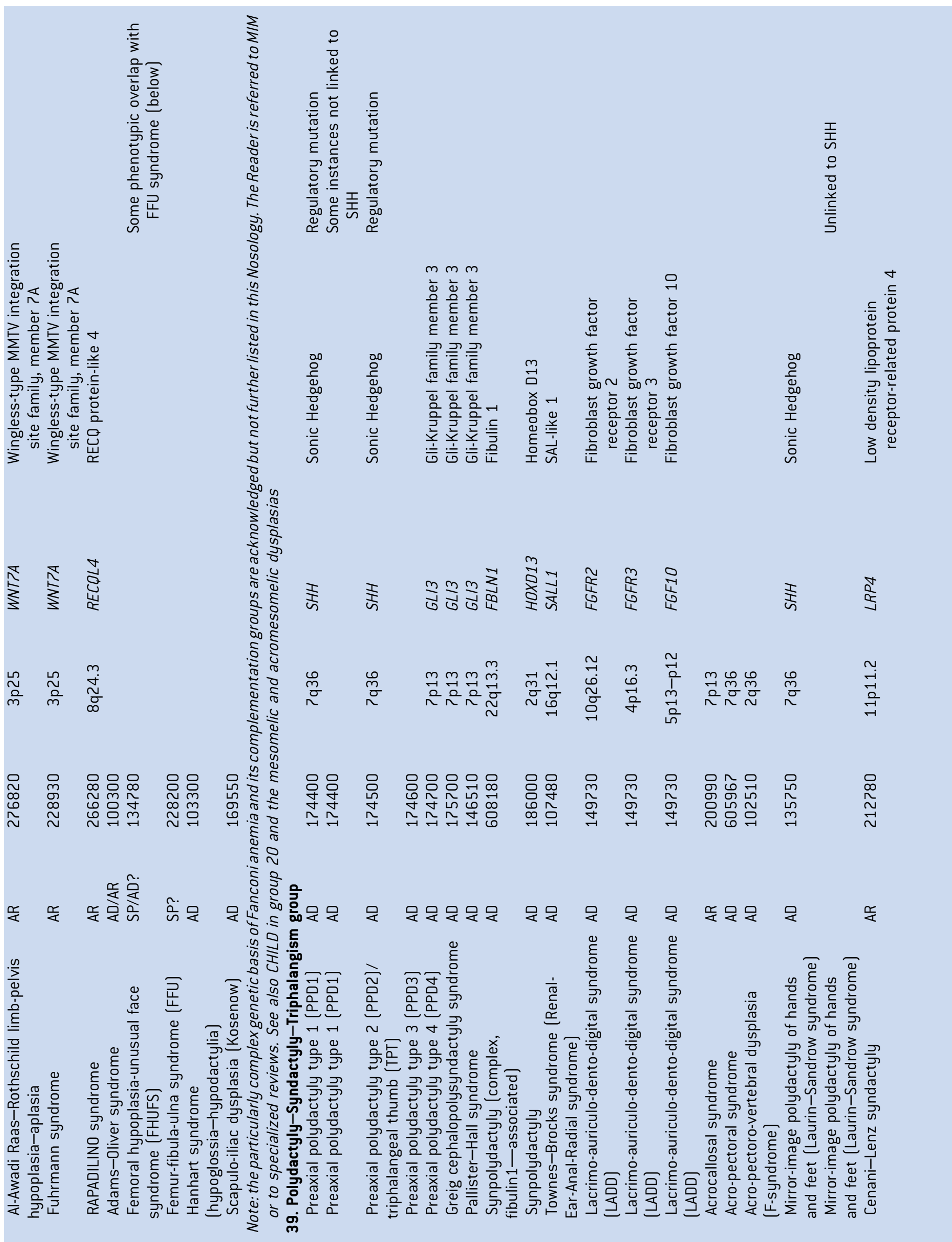




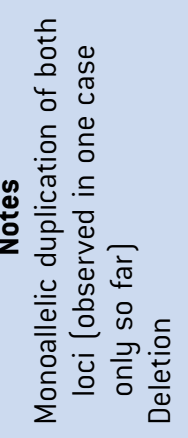

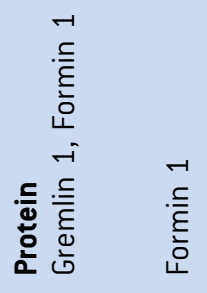
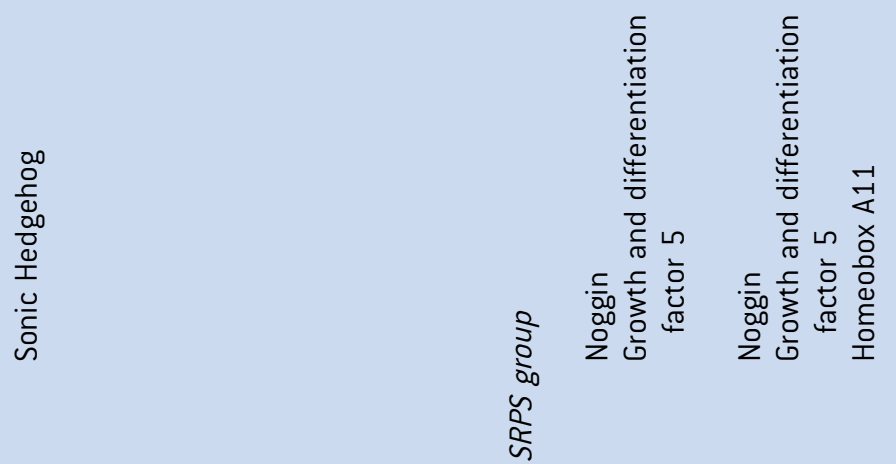

$\sum_{i=1}^{-1}$

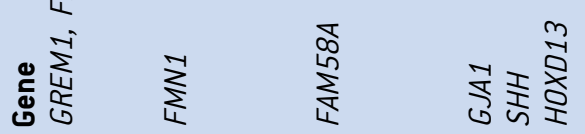

ஸิ ஃ

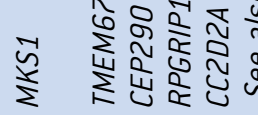

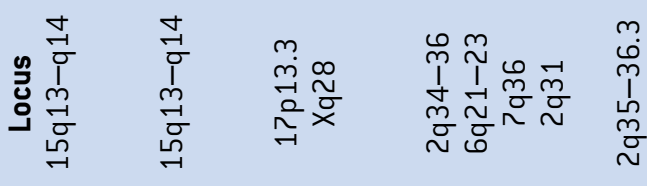

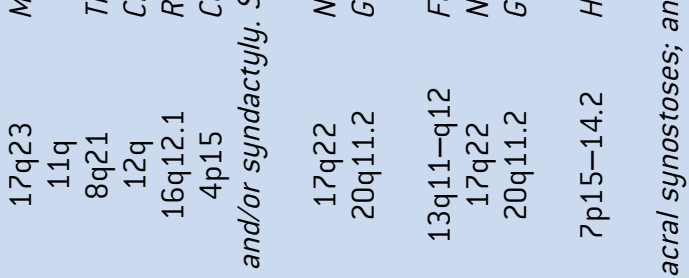

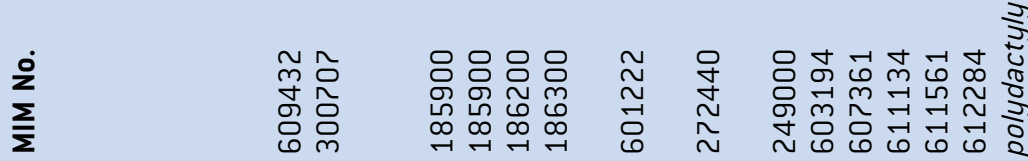
ST)

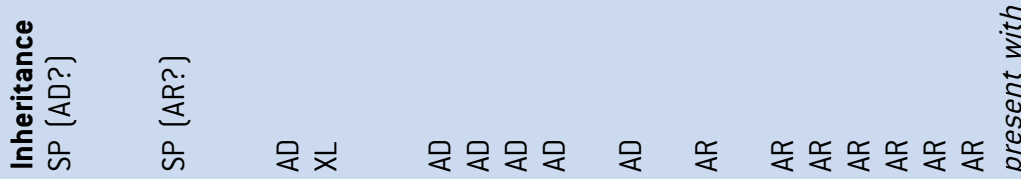

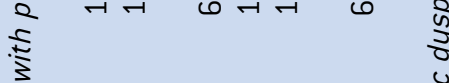

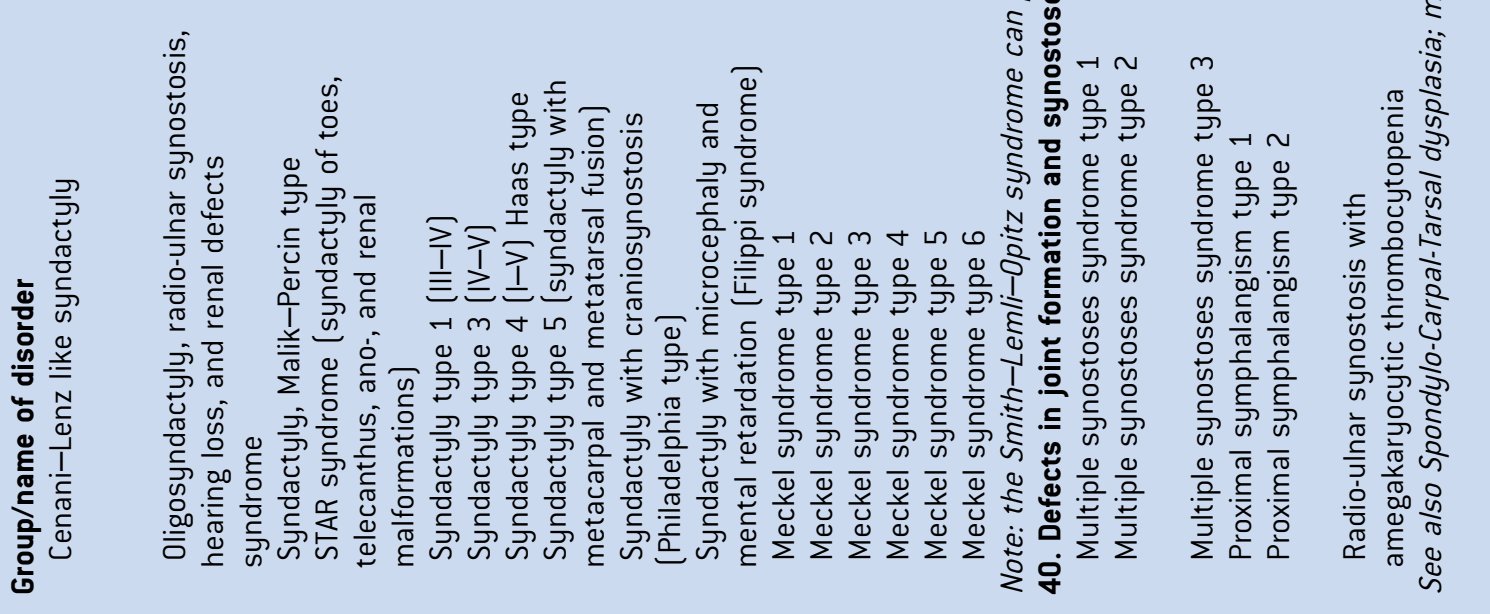


each gene separately, advocated by some scholars, is more confusing than helpful in clinical practice.

Group 26 has seen the identification of several novel molecular mechanisms leading to hypophosphatemic rickets.

In Group 29 (Disorganized Development of Skeletal Components), neurofibromatosis type 1 has been included following the points made by Stevenson and others that although the main clinical features of NF1 are neurologic and cutaneous, the skeletal features are frequent, diagnostically helpful and clinically relevant [Stevenson et al., 2007].

Groups 30 (Overgrowth syndromes with significant skeletal involvement) and Group 31 (Genetic inflammatory/rheumatoidlike osteoarthropathies) have been newly added. Group 30 comprises disorders that present as overgrowth syndromes and have a significant skeletal component that is part of the diagnostic criteria for a specific condition. One condition has been tentatively included because of its conspicuous skeletal features [Nishimura et al., 2004; Schmidt et al., 2007]; however this condition remains incompletely delineated. Group 31 includes disorders with features of inflammation and skeletal involvement. The creation of these two groups has been suggested by the frequent diagnostic overlap between these disorders and primary skeletal disorders as well as by the identification of the genetic basis of such disorders in recent years, allowing for a more precise delineation of the phenotypes.

Finally, groups 32-40 are dedicated to the dysostoses and follow again anatomical criteria (cranium, face, axial skeleton, extremities) with additional criteria reflecting principles of embryonic development such as limb reduction or hypoplasia (proximaldistal growth) versus terminal differentiation and patterning of the digits or joint formation. These groups have seen a marked increase in conditions with identified molecular bases and there are indications of a much larger heterogeneity yet.

A single group, the Brachyolmias (formerly group 13), has been deleted. Following the inclusion of dominant brachyolmia in the TRPV4 group, the few remaining short-trunk disorders have been incorporated in the SED group.

\section{DISCUSSION}

\section{Why "Groups"?}

The assignment of individual disorders into groups has been practiced since the first versions of the "Nomenclature." At that time, with little biochemical or molecular information available, the grouping of disorders reflected the belief that disorders with similar phenotypic features (e.g., dysostosis multiplex) might be caused by disturbances in related metabolic pathways or gene networks (in the case of dysostosis multiplex, lysosomal degradation). This notion has been confirmed by the identification of biochemically related groups, such as those of mineralization disorders or lysosomal disorders, and of genetic families such as the collagen 2 family, the FGFR3 family, and the DTDST family. The grouping of disorders is necessary because of the sheer number of conditions included, and can be helpful in making a differential diagnosis based on the main phenotypic findings, for example, in the mesomelic dysplasias or in chondrodysplasia punctata. Some groups are still defined by common radiographic features or by anatomical site involved. More- over, the nosology committee recognizes that some readers may disagree with our placement of a clinical entity into one group, when it may fit equally well in another group.

\section{Which Classification Criteria to Use?}

Criticism to the previous versions of the Nosology has focused on its "hybrid" nature, in the sense that it does not stick to a single systematic approach, be it clinical or molecular. This hybrid nature is intrinsic to the process of unraveling the underlying bases of skeletal diseases; disorders are classified on phenotypic similarities first, and as their molecular bases become understood they may be reclassified based on the gene or pathway that is abnormal. The first aim of the Nosology is to provide a reference list, and only secondarily to help in the diagnostic process. It must therefore coexist with other classifications that are based either on the clinical and radiographic approach to diagnosis, or the affected molecular systems and pathways. As more and more resources are published on the World Wide Web, crosslinking between classifications and databases may facilitate their simultaneous use.

Although care has been given to apply the inclusion criteria uniformly, there are disorders without proven molecular or biochemical defect for which inclusion in the Nosology as distinct entities seem somewhat arbitrary. For these disorders, discussion within the Nosology group, where individual opinions can be harmonized and, if needed, corrected by the collective expertise, is of great importance. Moreover, there are disorders listed in MIM that have not met our inclusion criteria, in most instances because of too few observations or because of the lack of features allowing clear diagnostic distinction from other disorders. It is likely that additional observations or the demonstration of a distinct molecular basis will allow for the inclusion of many of these disorders in the future, either as separate entities or as "variants" of already existing ones.

\section{Dysplasias Versus Dysostoses}

Dysostoses are disorders affecting individual bones or group of bones. In contrast to the "dysplasias," that arise frequently from defects in structural proteins, metabolic processes or in growth plate regulation, the dysostoses often arise from embryonic morphogenic defects and are thus more closely related to multiple malformation syndromes. Since the first inclusion of dysostoses in the 2001 revision, the number of "dysostoses" included in the Nosology has grown significantly. The present revision includes an even larger number of dysostoses reflecting the advances made in identifying their molecular basis. The boundaries between skeletal dysplasias and dysostoses, metabolic and molecular disorders, and multiple congenital anomalies syndromes is becoming progressively less sharp, and the diagnostic process requires knowledge that crosses between these subspecialty areas; the group of (craniofrontonasal disorders and the Franck-ter Haar syndrome can be cited as examples. The MIM catalogue contains many more entries, such as multiple malformation syndromes, that have some degree of skeletal involvement. Emphasis has been given to syndromes in which the skeletal component is prominent and/or essential to the diagnosis. 


\section{OMIM and the Nosology}

Because of the importance of consistency between parallel databases, the relationship between the Nosology and the OMIM database has been reviewed. The more comprehensive nature of the data collection and filing in MIM and the different nature of its revision process can lead to a divergence between the inclusion of nosologic entities and their denomination. Thus, MIM is in general more appositional, while the Nosology tries to do some "housekeeping" of entities by regrouping them and by eliminating those that have been incorporated into others. Efforts to made to harmonize the MIM and the Nosology are underway.

\section{Outlook}

The increasing availability of massive parallel sequencing and other new sequencing technologies will likely result in a rapid identification of novel disease-causing genes, but also in novel phenotypes associated with mutations in genes already linked to other phenotypes. In the near future, the catalog of skeletal phenotypes with a genetic basis may become so large as to surpass the scope of a "Nosology" as we understand it presently, and the Nosology will transform into an annotated database.

Even in that case, the many revisions of the Nosology will hopefully have paved the way by setting standards for the recognition and definition of skeletal phenotypes. Past versions of the Nosology have been translated in different languages and have found their way into textbooks of pediatrics and genetics. At present, the Nosology may help the clinician who is struggling for a diagnosis, by providing a simple listing of disorders grouped by cardinal features. The Nosology offers a quick reminder of the many differential diagnoses for one given disorder. As an expert-reviewed list of currently recognized disorders, the Nosology also constitutes a standard against which a possible "new" disorder should be compared. Finally, the Nosology offers a catalogue of genes involved in skeletal development and homeostasis that will be of interest and of inspiration to all those who are working in skeletal biology and medicine.

\section{ACKNOWLEDGMENTS}

M.L.W. is an Investigator with the Howard Hughes Medical Institute, and A.S.F. is supported by the Leenaards Foundation (Lausanne, Switzerland) and by the Faculté de Biologie et Medicine of the Lausanne University.

\section{REFERENCES}

1970. International nomenclature of constitutional diseases of bones. Ann Radiol (Paris) 13:455-464. 1971a. A nomenclature for constitutional (intrinsic) diseases of bones. J Pediatr 78:177-179.

1971b. International nomenclature of constitutional bone diseases. Constitutional bone diseases without known pathogenesis. Arch Fr Pediatr 28:553-557.

1971c. Nomenclature for constitutional (intrinsic) diseases of bones. Pediatrics 47:431-434.

1971d. Nomenclature for the constitutional (intrinsic) diseases of bone. Radiology 99:699-702.

1978. International nomenclature of constitutional diseases of bone. Revision-May, 1977. J Pediatr 93:614-616.

1979. International nomenclature of constitutional diseases of bone: Revision-May 1977. Am J Med Genet 3:21-26.

1983. International nomenclature of constitutional diseases of bone. Revision, May, 1983. Ann Radiol (Paris) 26:457-462.

1998. International nomenclature and classification of the osteochondrodysplasias (1997). International working group on constitutional diseases of bone. Am J Med Genet 79:376-382.

Hall CM. 2002. International nosology and classification of constitutional disorders of bone (2001). Am J Med Genet 113:65-77.

Lachman RS. 1998. International nomenclature and classification of the osteochondrodysplasias (1997). Pediatr Radiol 28:737-744.

McKusick VA, Scott CI. 1971. A nomenclature for constitutional disorders of bone. J Bone Joint Surg Am 53:978-986.

Nishimura G, Hasegawa T, Kinoshita E, Tanaka Y, Kurosawa K, Yoshimoto M. 2004. Newly recognized syndrome of metaphyseal undermodeling, spondylar dysplasia, and overgrowth: Report of two adolescents and a child. Am J Med Genet Part A 128A:204-208.

Rimoin DL. 1979. International nomenclature of constitutional diseases of bone with bibliography. Birth Defects Orig Artic Ser 15:30.

Schmidt H, Kammer B, Grasser M, Enders A, Rost I, Kiess W. 2007. Endochondral gigantism: A newly recognized skeletal dysplasia with preand postnatal overgrowth and endocrine abnormalities. Am J Med Genet Part A 143A:1868-1875.

Sillence DO, Rimoin DL. 1978. Classification of osteogenesis imperfect. Lancet 1:1041-1042.

Sillence DO, Rimoin DL, Danks DM. 1979a. Clinical variability in osteogenesis imperfecta-variable expressivity or genetic heterogeneity. Birth Defects Orig Artic Ser 15:113-129.

Sillence DO, Senn A, Danks DM. 1979b. Genetic heterogeneity in osteogenesis imperfecta. J Med Genet 16:101-116.

Spranger J. 1992. International classification of osteochondrodysplasias. The international working group on constitutional diseases of bone. Eur J Pediatr 151:407-415.

Stevenson DA, Viskochil DH, Carey JC. 2007. Neurofibromatosis type 1 is a genetic skeletal disorder. Am J Med Genet Part A 143A:2082-2083; author reply 2084.

Superti-Furga A, Unger S. 2007. Nosology and classification of genetic skeletal disorders: 2006 revision. Am J Med Genet Part A 143A:1-18. 


\section{University Library}

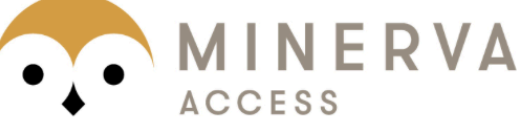

A gateway to Melbourne's research publications

Minerva Access is the Institutional Repository of The University of Melbourne

\section{Author/s:}

Warman, ML;Cormier-Daire, V;Hall, C;Krakow, D;Lachman, R;LeMerrer, M;Mortier, G;Mundlos, S;Nishimura, G;Rimoin, DL;Robertson, S;Savarirayan, R;Sillence, D;Spranger, J;Unger, S;Zabel, B;Superti-Furga, A

Title:

Nosology and Classification of Genetic Skeletal Disorders: 2010 Revision

Date:

2011-05-01

Citation:

Warman, M. L., Cormier-Daire, V., Hall, C., Krakow, D., Lachman, R., LeMerrer, M., Mortier, G., Mundlos, S., Nishimura, G., Rimoin, D. L., Robertson, S., Savarirayan, R., Sillence, D., Spranger, J., Unger, S., Zabel, B. \& Superti-Furga, A. (2011). Nosology and Classification of Genetic Skeletal Disorders: 2010 Revision. AMERICAN JOURNAL OF MEDICAL GENETICS PART A, 155A (5), pp.943-968. https://doi.org/10.1002/ajmg.a.33909.

Persistent Link:

http://hdl.handle.net/11343/264780

License:

CC BY 